\title{
THE HERSCHEL* EXPLOITATION OF LOCAL GALAXY ANDROMEDA (HELGA). VI. THE DISTRIBUTION AND PROPERTIES OF MOLECULAR CLOUD ASSOCIATIONS IN M31
}

\author{
J. M. KirK ${ }^{1,2}$, W. K. Gear ${ }^{2}$, J. Fritz ${ }^{3}$, M. W. L. Smith ${ }^{2}$, G. Ford², M. Baes ${ }^{3}$, G. J. Bendo ${ }^{4}$, I. De Looze ${ }^{3}$, S. A. Eales ${ }^{2}$,

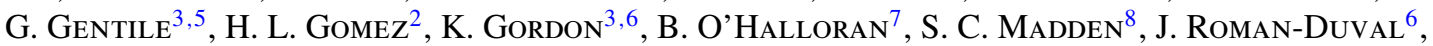 \\ J. VerstapPen ${ }^{3,9}$, S. Viaene ${ }^{3}$, A. Boselli ${ }^{10}$, A. Cooray ${ }^{11}$, V. Lebouteiller ${ }^{8}$, And L. Spinoglio ${ }^{12}$ \\ ${ }^{1}$ Jeremiah Horrocks Institute, University of Central Lancashire, Preston PR1 2HE, UK \\ ${ }^{2}$ School of Physics and Astronomy, Cardiff University, Queens Buildings, The Parade, Cardiff, Wales CF24 3AA, UK \\ ${ }^{3}$ Sterrenkundig Observatorium, Universiteit Gent, Krijgslaan 281 S9, B-9000 Gent, Belgium \\ ${ }^{4}$ UK ALMA Regional Centre Node, Jodrell Bank Centre for Astrophysics, School of Physics and Astronomy, University of Manchester, \\ Oxford Road, Manchester M13 9PL, UK \\ ${ }^{5}$ Department of Physics and Astrophysics, Vrije Universiteit Brussel, Pleinlaan 2, B-1050 Brussels, Belgium \\ ${ }^{6}$ Space Telescope Science Institute, 3700 San Martin Drive, Baltimore, MD 21218, USA \\ ${ }^{7}$ Astrophysics Group, Imperial College, Blackett Laboratory, Prince Consort Road, London SW7 2AZ, UK \\ ${ }^{8}$ Laboratoire AIM, CEA/DSM-CNRS-Université Paris Diderot, Irfu/Service, Paris, F-91190 Gif-sur-Yvette, France \\ ${ }^{9}$ Kapteyn Astronomical Institute, P.O. Box 800, 9700 AV Groningen, The Netherlands \\ ${ }^{10}$ Laboratoire d'Astrophysique de Marseille, UMR 7326 CNRS, 38 rue F. Joliot-Curie, F-13388 Marseille, France \\ ${ }^{11}$ Department of Physics and Astronomy, University of California, Irvine, CA 92697, USA \\ 12 Istituto di Astrofisica e Planetologia Spaziali (INAF-IAPS), via del Fosso del Cavaliere 100, Roma I-00133, Italy \\ Received 2013 June 12; accepted 2014 September 10; published 2014 December 19
}

\begin{abstract}
In this paper we present a catalog of giant molecular clouds (GMCs) in the Andromeda (M31) galaxy extracted from the Herschel Exploitation of Local Galaxy Andromeda (HELGA) data set. GMCs are identified from the Herschel maps using a hierarchical source extraction algorithm. We present the results of this new catalog and characterize the spatial distribution and spectral energy properties of its clouds based on the radial dust/gas properties found by Smith et al. A total of 326 GMCs in the mass range $10^{4}-10^{7} M_{\odot}$ are identified; their cumulative mass distribution is found to be proportional to $M^{-2.34}$, in agreement with earlier studies. The GMCs appear to follow the same correlation of cloud mass to $L_{\mathrm{CO}}$ observed in the Milky Way. However, comparison between this catalog and interferometry studies also shows that the GMCs are substructured below the Herschel resolution limit, suggesting that we are observing associations of GMCs. Following Gordon et al., we study the spatial structure of M31 by splitting the observed structure into a set of spiral arms and offset rings. We fit radii of 10.3 and $15.5 \mathrm{kpc}$ to the two most prominent rings. We then fit a logarithmic spiral with a pitch angle of 8.9 to the GMCs not associated with either ring. Last, we comment on the effects of deprojection on our results and investigate the effect different models for M31's inclination will have on the projection of an unperturbed spiral arm system.
\end{abstract}

Key words: galaxies: individual (M31) - galaxies: ISM - galaxies: structure - ISM: clouds

Supporting material: machine-readable tables

\section{INTRODUCTION}

The study of star formation within our own Galaxy is limited by our ability to resolve molecular clouds from the tangled web of the Galactic disk. The disk of the Milky Way has been mapped as part of the CO survey of Dame et al. (2001) and the Spitzer GLIMPSE (Churchwell et al. 2009) and MIPSGAL surveys (Carey et al. 2009), among others, most recently by the Hi-GAL Herschel Open Time Key Project (Molinari et al. 2010). Nevertheless, ensemble studies of giant molecular clouds (GMCs) within our own Galaxy are still hampered by distance ambiguities and sampling limitations imposed by our own position within the Galactic disk.

The solution to this is to study the same molecular clouds in nearby galaxies where we can observe the entire disk. The nearest spiral galaxy $(785 \pm 25 \mathrm{kpc}$, McConnachie et al. 2005) to our own Milky Way is the Andromeda galaxy (M31). It is the largest member of the Local Group of galaxies, of which our own Milky Way is the second largest. Herschel Exploitation of Local Galaxy Andromeda (HELGA; Fritz et al. 2012, hereafter

\footnotetext{
* Herschel is an ESA space observatory with science instruments provided by European-led Principal Investigator consortia and with important participation from NASA.
}

Paper I) is the first comprehensive, high-resolution, far-infrared (FIR) and submillimeter survey of M31 and its surroundings. There is a long-established link between molecular gas emission and observations of dust (e.g., Burstein \& Heiles 1982), so the Herschel data set allows us to trace emission from the GMCs in M31.

GMCs were first cataloged in the Milky Way as dark nebulae, dust silhouettes seen against a bright background star field (Barnard 1919; Lynds 1962), but a comparable catalog of 730 dark nebulae in M31 was not published until the 1980s (Hodge 1980). Single-dish CO observations mapped the molecular gas associated with these dark nebulae (Boulanger et al. 1981; Lada et al. 1988), but it was not until the first small interferometer maps were produced (Vogel et al. 1987; Wilson \& Rudolph 1993; Allen et al. 1995; Loinard \& Allen 1998) that individual GMCs could be resolved. Later studies were able to cover larger areas as Sheth et al. (2000, 2008, hereafter S08) mapped six clouds and Rosolowsky (2007, hereafter R07) mapped 67 clouds. R07 was the first study to perform a statistical analysis of GMCs in M31. Many of these studies (e.g., Vogel et al. 1987, R07, S08) have noted that individually or in assemblage, M31's GMCs resemble those in the Milky Way. The HELGA data now allow us to determine whether this correspondence extends to the entire disk of M31. 


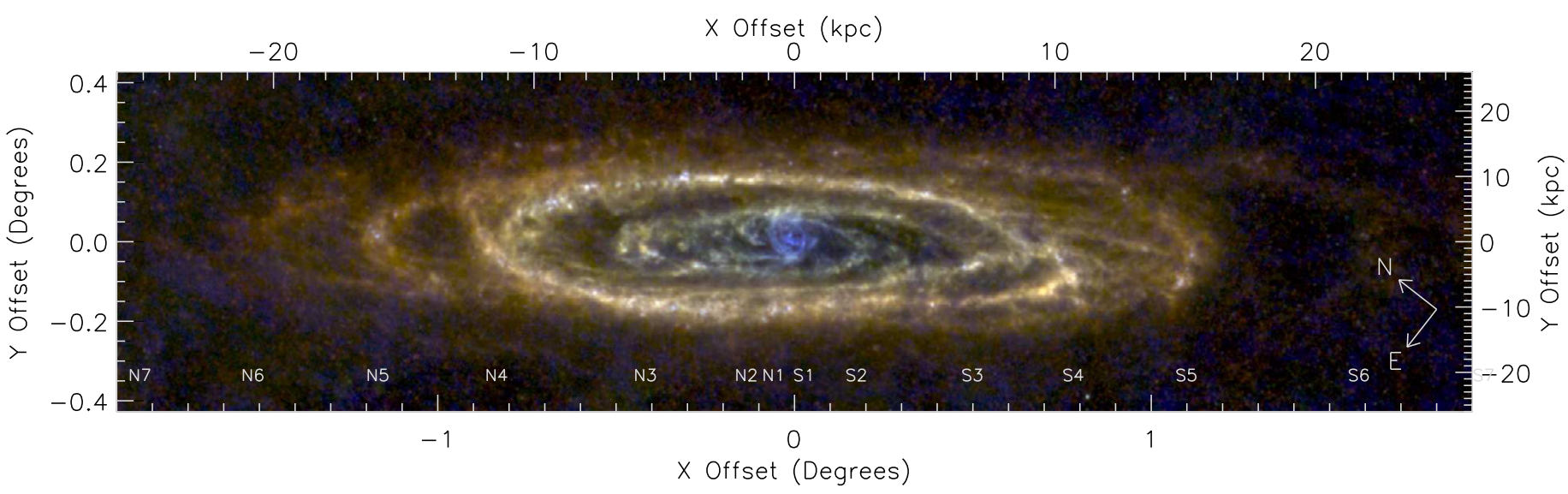

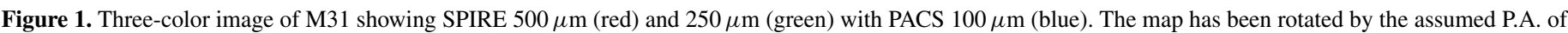

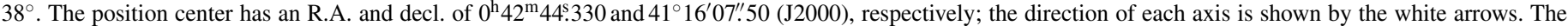

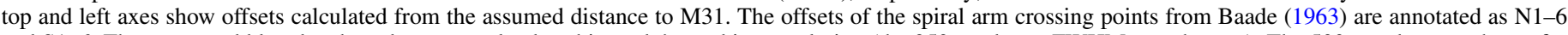

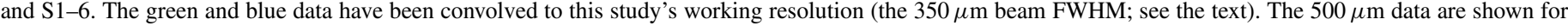
comparison only and are left unconvolved.

The dominant feature of M31 is a $100^{\prime}$ diameter ring that appears in continuum emission from the infrared (Habing et al. 1984; Haas et al. 1998; Gordon et al. 2006) to the radio (Bystedt et al. 1984). The ring is also detected in $\mathrm{H} \alpha$ (Arp 1964; Devereux et al. 1994) and carbon monoxide (Nieten et al. 2006). At the usual distance estimates to Andromeda, the ring has a radius of $10 \mathrm{kpc}$. Gordon et al. (2006) used deprojected Spitzer $24 \mu \mathrm{m}$ data to fit a circle of radius $9.8 \mathrm{kpc}$ to the ring and showed that its center was offset from the center of the spiral arm pattern. In Paper I we showed how a comparison of Herschel and $\mathrm{H}$ I atomic data revealed the presence of several low-intensity extended features we named $\mathrm{E}, \mathrm{F}$, and $\mathrm{G}$ at radii of 21,26 , and $31 \mathrm{kpc}$, respectively. These features appear to form additional rings or arms beyond the well-known $10 \mathrm{kpc}$ ring and the fainter $15 \mathrm{kpc}$ ring (Haas et al. 1998; Gordon et al. 2006).

A key factor in deriving parameters from FIR observations is a practical knowledge of the dust grain properties. In Smith et al. (2012, hereafter Paper II) we compared the HELGA data to the molecular gas as traced by carbon monoxide line maps in order to examine the effects of metallicity gradients on the dust-togas ratio across the M31 disk. It was found that the gas-to-dust ratio $r_{\mathrm{gd}}$ had an exponential dependence with radius of the form $\log r_{\mathrm{gd}}=1.1+0.0496 R$, where $R$ is the galactocentric radius. Paper I found that the dust emissivity index, $\beta$, was $\sim 1.9$ in the $10 \mathrm{kpc}$ ring, in broad agreement with studies of local Milky Way clouds (e.g., Planck Collaboration et al. 2011). However, Paper II also demonstrated that $\beta$ varied globally from a high of $\sim 2.5$ in the center to a value of $\sim 1.7$ at large radii.

Ford et al. (2013, hereafter Paper III) combined Galex farUV and Spitzer $24 \mu \mathrm{m}$ data sets to make a star formation rate (SFR) map of M31 and found a global SFR of $0.25 M_{\odot} \mathrm{yr}^{-1}$. This rate is a quarter of that of the Milky Way (Robitaille \& Whitney 2010; Lee et al. 2012), despite their masses as inferred from the motion of their satellites being comparable (Watkins et al. 2010; Reid et al. 2009). Paper III also showed that M31 was positioned below the scatter of normalspiral galaxies on the Kennicutt-Schmidt plot (Schmidt 1959; Kennicutt 1998b) of mass versus SFR surface densities. One of the questions that arises from these studies is whether the differences in SFR between M31 and the Milky Way are due to a difference in the number of GMCs (the sites of star formation) or to a fundamental difference in the individual GMCs' properties.
In this paper we use the HELGA data to analyze the population of GMCs and GMC complexes in M31. In Section 2 we briefly describe the HELGA data, and in Section 3 we describe the source extraction technique using the CSAR (Conservative Source AlgoRithm) dendrogram algorithm (Kirk et al. 2013). In Section 4 we examine the properties of the extracted sources and compare them with the observations of clouds from the Milky Way. Then in Section 5 we reexamine the structure of M31 based on the positions of the Herschel sources.

\section{OBSERVATIONS}

M31 was observed on 2010 December 18-20 and 2011 January 23 (Observation Days 584-586 and 620) using the parallel mode of the SPIRE (Griffin et al. 2010) and PACS (Poglitsch et al. 2010) cameras aboard the Herschel Space Observatory (Pilbratt et al. 2010). These data used PACS filters centered at wavelengths of $100 \mu \mathrm{m}$ and $160 \mu \mathrm{m}$, with angular resolutions of $12^{\prime \prime} .5$ and $13^{\prime \prime} .3$, respectively (accounting for the scan speed of $60^{\prime \prime} \mathrm{s}^{-1}$ ), and SPIRE filters centered at wavelengths of $250 \mu \mathrm{m}$, $350 \mu \mathrm{m}$, and $500 \mu \mathrm{m}$, with angular resolutions of $18^{\prime \prime} .2,24^{\prime \prime} .5$, and 36.'0, respectively. The observation strategy and data reduction methods are described in detail in Paper I. No Planck calibration offsets were applied (e.g., Bernard et al. 2010), as we will only be performing background-subtracted (i.e., DC level removed) photometry.

Figure 1 shows a three-color image of M31 using SPIRE $500 \mu \mathrm{m}$ (red), $250 \mu \mathrm{m}$ (green), and PACS $100 \mu \mathrm{m}$ (blue) in rotated coordinates. We use a position center with a right ascension and declination of $00^{\mathrm{h}} 42^{\mathrm{m}} 44.330$ and $41^{\circ} 16^{\prime} 07^{\prime \prime} .50$, respectively (Skrutskie et al. 2006, the 2MASS catalog position). In common with other HELGA studies, we assume a distance to Andromeda of $785 \pm 25 \mathrm{kpc}$ (McConnachie et al. 2005) and global inclination and P.A.s of $77^{\circ}$ and $38^{\circ}$, respectively. A discussion of the assumed angles is included in Appendix A. At this distance, the final PACS and SPIRE angular resolutions are equivalent to spatial resolutions of $48-137 \mathrm{pc}$.

The dominant feature at the longer wavelengths is the $10 \mathrm{kpc}$ ring. This ring is seen in Figure 1 as the yellow/white loop traced by strong SPIRE emission. Also visible in Figure 1 is the color difference between the cooler ring and the bluer, warmer galaxy center. 


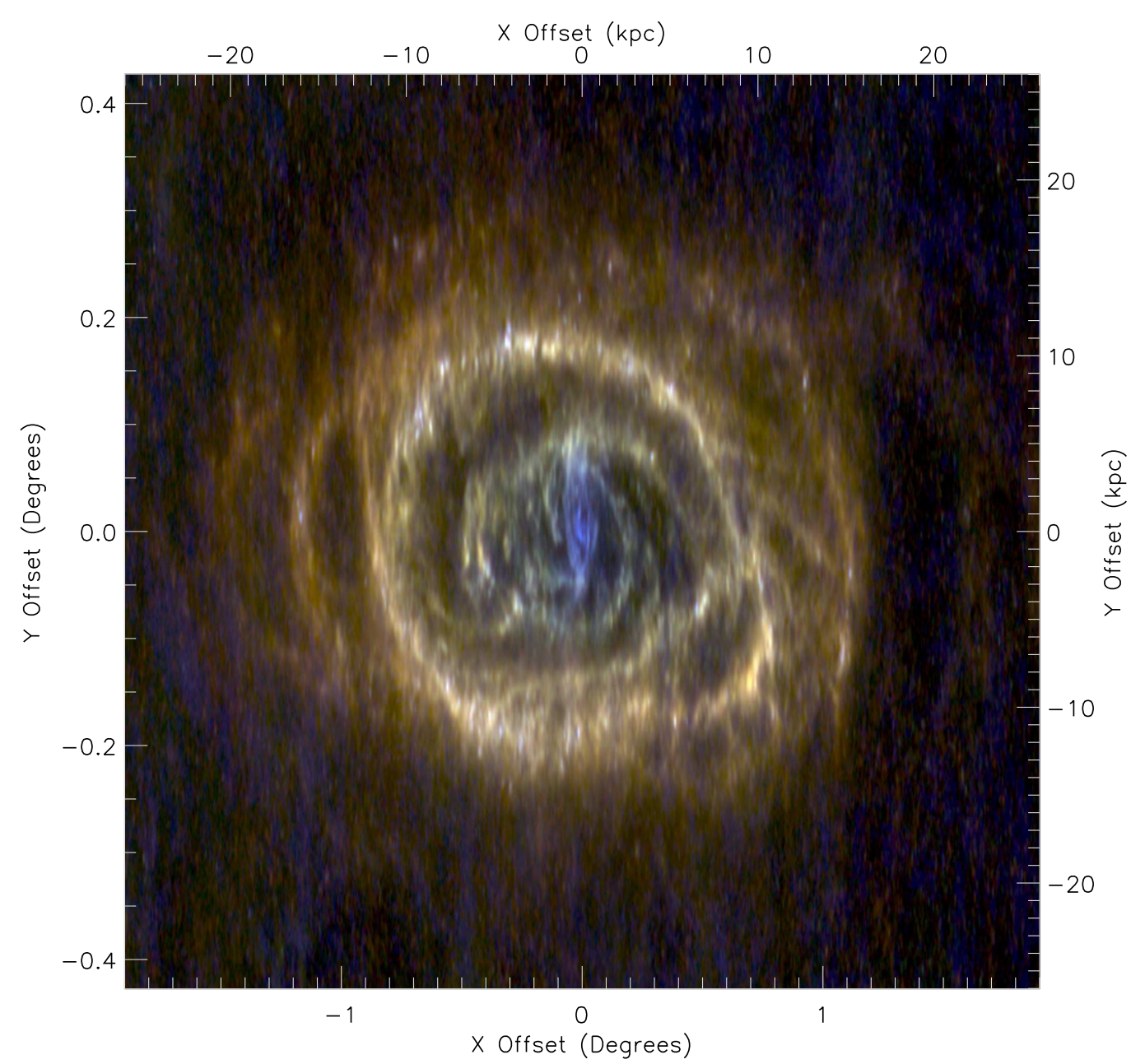

Figure 2. Same as Figure 1, except that the $y$-axis has been deprojected using the assumed inclination angle of $77^{\circ}$.

Andromeda is highly inclined and displays a significant warp at larger radii (e.g., Chemin et al. 2009; Corbelli et al. 2010). The radius of the disk viewed in the Herschel images (e.g., Figure 1) is $\sim 20 \mathrm{kpc}$ (similar to the radius out to which we can extract sources; see Figure 5), approximately half that for which H I models have been computed (e.g., Chemin et al. 2009; Corbelli et al. 2010). Figure 2 shows the same as Figure 1 except with the $y$-axis deprojected using the assumed inclination angle of $77^{\circ}$. We discuss the magnitude of the discrepancy between the assumption of flat and warped geometry on the deprojected structure in Appendix A.

\section{CATALOG}

\subsection{Source Identification}

The emission from M31, as with any spiral galaxy, is highly hierarchical and is organized into tiers of rings, arms, complexes, and clouds. A source extraction algorithm must take this tree, the hierarchy of nested structures, into account. For this purpose we use the CSAR source extraction algorithm (Kirk et al. 2013). CSAR works by processing the pixels in an image in order of descending flux, assigning each pixel in turn to a region centered on a local maximum. A region is flagged as significant if it passes contrast (signal-to-noise ratio $[\mathrm{S} / \mathrm{N}]>5 \sigma$ ) and size (larger than the telescope point-spread function [PSF]) criteria. Neighboring regions grow downward in flux until they touch. If both regions are significant, then a record of their state is made.
The regions are then merged. The process continues until all pixels above a minimum value have been processed.

CSAR is, in effect, walking through the binary structure tree of the map that it is being run on. The tree is made from nodes, in effect single closed isophotal contours, and branches that relate how one node nests (encloses) another pair of nodes (contours). Nodes at the very top of the tree, the leaf nodes, contain no other nodes-i.e., they contain no resolved substructure-and are directly analogous to a normal source. At the base of the tree is the root node; this is the node that contains all the other nodes. Thus, the tree describes an entire region as a set of closed isophotal contours and defines the region's structure by recording how those contours nest within each other.

While the utility of using structure tree decomposition to study the hierarchical properties of molecular clouds has been known for some time (Houlahan \& Scalo 1992), its practical application has only recently become routine (Rosolowsky et al. 2008; Goodman et al. 2009; Wünsch et al. 2012). The same theory has also been applied to the hierarchical relationship of stellar clusters in Local Group galaxies (Gouliermis et al. 2010). The theory behind the Rosolowsky et al. (2008) and Wünsch et al. (2012) codes and CSAR is similar, although CSAR is designed to work with monochromatic data.

The $350 \mu \mathrm{m}$ SPIRE image was used to define the working resolution/pixel grid as it improves on the resolution of the $500 \mu \mathrm{m}$ data and still leaves at least three resolved data points across the spectral energy distribution (SED) peak (enough 


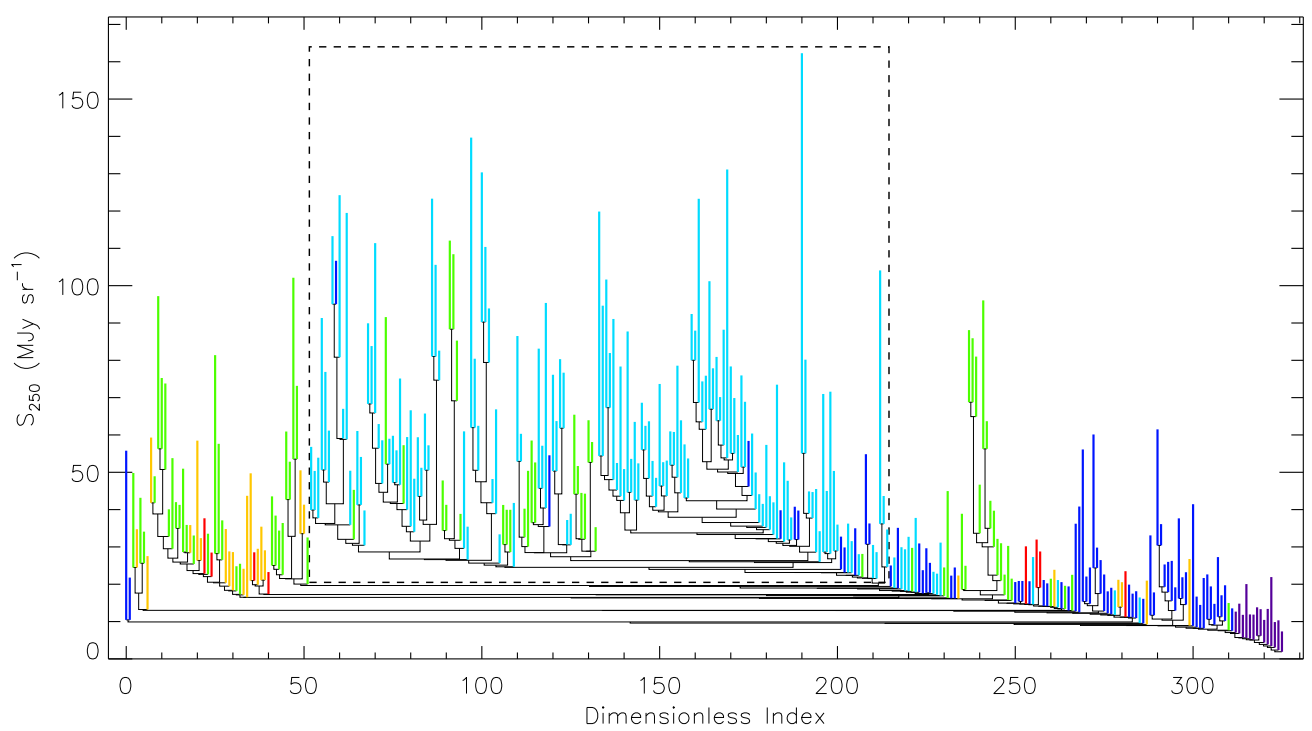

Figure 3. Dendrogram of $250 \mu \mathrm{m}$ intensity structure toward M31. The peak-to-background extent of each node is shown by the vertical bars. The colored bars are the leaf nodes; the color denotes the galactocentric radius of each node (see Figure 4). The horizontal lines show the equivalent contour level at which two neighboring nodes merge. The dashed box plotted over the dendrogram shows the branch containing the $10 \mathrm{kpc}$ ring.

points to fit a two-parameter SED against). All data were reprojected onto this grid for analysis (see Section 3.3). CSAR was run on the smoothed/resampled $250 \mu \mathrm{m}$ image of M31 as this was the channel with the highest $\mathrm{S} / \mathrm{N}$. The extraction was performed on the data before they were deprojected in order to avoid problems introduced by a noncircular PSF. M31 is surrounded by an extended noisy structure that does not appear to be coherent (see Paper I); this is foreground Galactic cirrus. This cirrus is particularly visible in the northeast corner of the M31 field and is distinct from M31 in H I line channel maps (see Figure 4 of Paper I). We exclude the cirrus from our analysis by pruning the tree back to the branch that we know just contains M31 emission.

\subsection{Dendrogram}

After pruning, the M31 tree was left with 651 nodes, of which 326 were leaf nodes. Figures 3 and 4 show the CSAR results for the M31 branch of the $250 \mu \mathrm{m}$ structure tree. Figure 3 shows a dendrogram (a form of tree diagram used to represent structures in hierarchical data sets) of the extracted structure within M31. The vertical axis is the $250 \mu \mathrm{m}$ intensity; the horizontal axis is an arbitrary-dimensionless index given to each leaf node such as to unwrap the tree structure and clearly show the separate branches without them overlapping. The peak-to-background $250 \mu \mathrm{m}$ intensity of each node is shown by the vertical colored bars. The horizontal lines show the equivalent contour level at which two nodes merge. Figure 4 shows the positions of the extracted nodes plotted over a map of SPIRE $250 \mu \mathrm{m}$ intensity. The color of the vertical bars in Figure 3 and the annotations in Figure 4 are the same and show the distance of the leaf nodes from the center of M31. This is the same color scheme as used by Paper III and is shown by the bar in Figure 4.

A striking feature of the dendrogram in Figure 3 is that several large branches all merge at approximately the same intensity level; this is the $10 \mathrm{kpc}$ ring. These branches are shown by the dashed box plotted over the dendrogram. The spatial extent of the region, the node just before the ring closes, is shown by the single contour in Figure 4. We can now walk down the structure tree from the brightest source to the faintest. The structures that compose the ring (the cyan markers) form several distinct complexes, but all merge together into a single structure at approximately the same intensity level $\left(\sim 20 \mathrm{MJy} \mathrm{sr}^{-1}\right)$. Several prominent structures interior to the $10 \mathrm{kpc}$ ring (the green clouds) then merge with the tree before the exterior clouds (shown in blue and purple) connect. These exterior sources are distinct from background sources in that they are connected to M31 by contiguous emission.

\subsection{Measured Properties}

The truncated tree contains 326 leaf nodes. We assume that these Herschel $250 \mu \mathrm{m}$ identified sources, which, as stated, have no resolved substructure, are GMCs or associations of several GMCs, hereafter referred to simply as clouds. Table 1 lists the properties of the clouds. The first column lists the catalog number (a dimensionless index assigned during the plotting of the dendrogram; see below). All positions and sizes are calculated from the moments of each source's $250 \mu \mathrm{m}$ half-power contour. The second and third columns list the right ascension and declination of the centroid of the halfpower contour. The kiloparsec $X$ and $Y$ offsets in the rotated, deprojected frame $\left(\theta=38^{\circ}, i=77^{\circ}\right)$ are listed in Columns 4 and 5. Column 6 lists the galactocentric distance $R$ of the clouds. The positional accuracy is on the order of the pixel size as the map is Nyquist sampled. Using the $350 \mu \mathrm{m}$ pixel grid, this is 8 arcsec, which equates to $30 \mathrm{pc}$ along the un-deprojected $x$-axis and $\sim 140 \mathrm{pc}$ along the deprojected $y$-axis. Column 7 lists the geometric mean of the deconvolved FWHMs of each cloud.

The CSAR extraction produces a mask on the $350 \mu \mathrm{m}$ pixel grid for each cloud. Integrated flux densities are calculated by summing the pixels under each pixel mask at each wavelength. Before the fluxes were measured, the data were convolved to a common resolution (the SPIRE $350 \mu \mathrm{m}$ PSF, 24" FWHM) using the Aniano et al. (2011) convolution kernels and then co-aligned on the $350 \mu \mathrm{m}$ pixel grid ( $8^{\prime \prime}$ pixel width). We estimate the local pixel rms for each cloud by calculating the standard deviation of the pixels immediately adjoining its bounding contour. The level of this isocontour is subtracted as a background from the pixels interior to it before the flux summation is performed. Each cloud's spectrum is color-corrected using the standard 


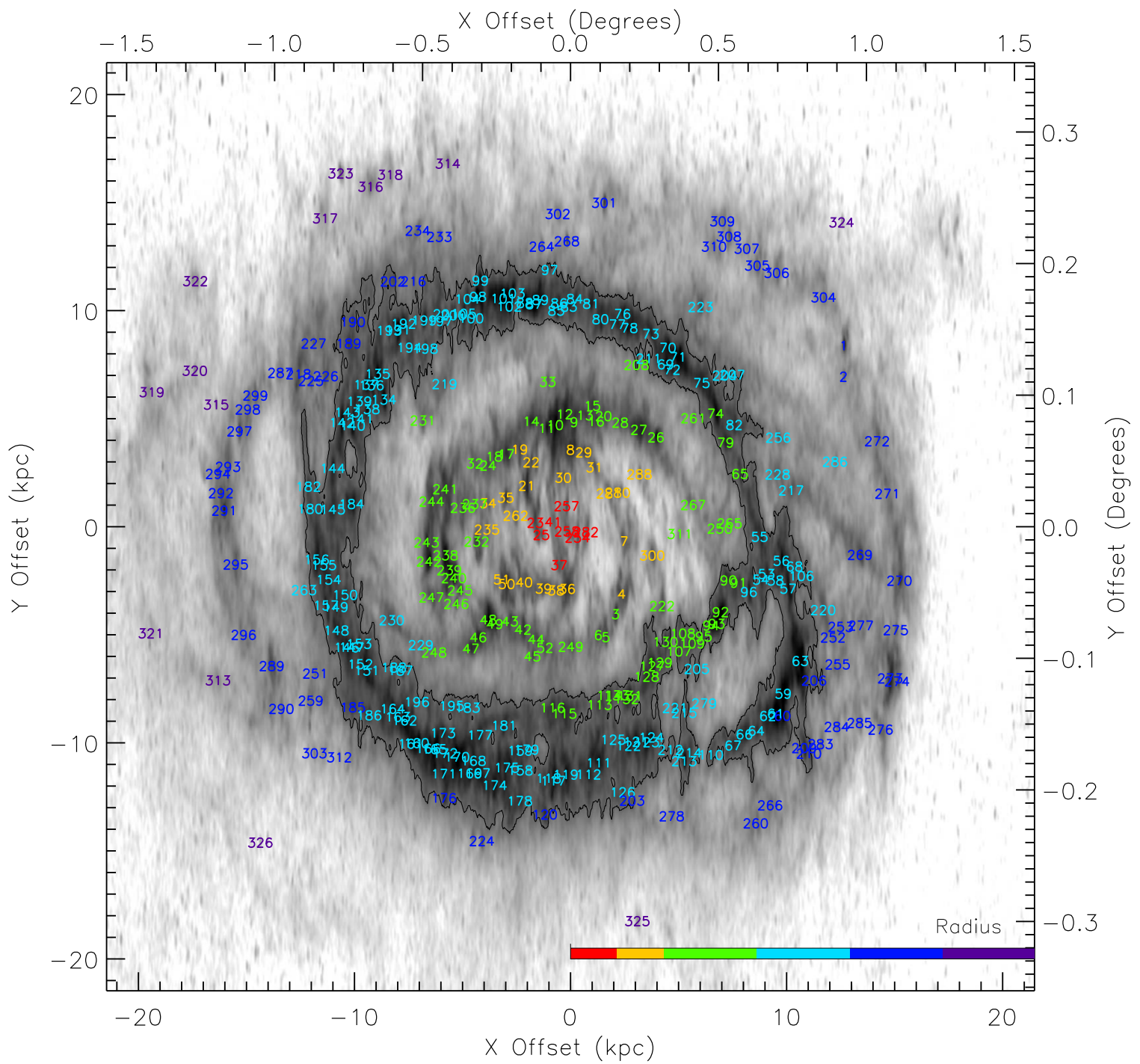

Figure 4. Relative positions of the leaf-node sources from Figure 3. The gray scale shows SPIRE $250 \mu \mathrm{m}$ intensity. The contour shows the area enclosing the $10 \mathrm{kpc}$ ring branch of the dendrogram. The axes show the offsets in kiloparsecs at the assumed distance to M 31 and in degrees. The leaf-node labels are colored depending on their galactocentric distance using the same color scheme as Paper III; the scheme is shown by the color bar.

Table 1

Table of Measured Leaf-node Parameters

\begin{tabular}{|c|c|c|c|c|c|c|c|c|c|c|}
\hline $\begin{array}{l}\text { Name } \\
\text { (HELGA) }\end{array}$ & $\begin{array}{l}\text { R.A. } \\
\text { (2000) }\end{array}$ & $\begin{array}{l}\text { Decl. } \\
\text { (2000) }\end{array}$ & $\begin{array}{c}X \\
(\mathrm{kpc})\end{array}$ & $\begin{array}{c}Y \\
(\mathrm{kpc})\end{array}$ & $\begin{array}{c}R \\
(\mathrm{kpc})\end{array}$ & $\begin{array}{c}\text { FWHM } \\
(\mathrm{pc})\end{array}$ & $\begin{array}{c}S_{350 \mu \mathrm{m}} \\
(\mathrm{Jy})\end{array}$ & $\begin{array}{c}S_{250 \mu \mathrm{m}} \\
(\mathrm{Jy})\end{array}$ & $\begin{array}{c}S_{160 \mu \mathrm{m}} \\
(\mathrm{Jy})\end{array}$ & $\begin{array}{c}S_{100 \mu \mathrm{m}} \\
(\mathrm{Jy})\end{array}$ \\
\hline 1 & $00^{\mathrm{h}} 39^{\mathrm{m}} 10^{\mathrm{s}} \cdot 2$ & $40^{\circ} 37^{\prime} 21^{\prime \prime}$ & 12.7 & 8.4 & 15.2 & 78 & $0.84 \pm 0.02$ & $1.8 \pm 0.1$ & $3.0 \pm 0.1$ & $2.1 \pm 0.1$ \\
\hline 2 & $00^{\mathrm{h}} 39^{\mathrm{m}} 16^{\mathrm{s}} .1$ & $40^{\circ} 36^{\prime} 30^{\prime \prime}$ & 12.6 & 7.0 & 14.4 & 78 & $0.27 \pm 0.02$ & $0.59 \pm 0.01$ & $0.76 \pm 0.07$ & $0.58 \pm 0.08$ \\
\hline 3 & $00^{\mathrm{h}} 42^{\mathrm{m}} 30^{\mathrm{s}} .9$ & $41^{\circ} 06^{\prime} 26^{\prime \prime}$ & 2.1 & -4.0 & 4.5 & 99 & $0.33 \pm 0.01$ & $0.81 \pm 0.03$ & $1.1 \pm 0.1$ & $0.47 \pm 0.04$ \\
\hline 4 & $00^{\mathrm{h}} 42^{\mathrm{m}} 22^{\mathrm{s}} .8$ & $41^{\circ} 05^{\prime} 59^{\prime \prime}$ & 2.4 & -3.1 & 3.9 & 220 & $0.31 \pm 0.02$ & $0.75 \pm 0.04$ & $0.97 \pm 0.07$ & $0.51 \pm 0.06$ \\
\hline 5 & $00^{\mathrm{h}} 42^{\mathrm{m}} 41^{\mathrm{s}} .6$ & $41^{\circ} 07^{\prime} 20^{\prime \prime}$ & 1.7 & -5.1 & 5.3 & 78 & $0.25 \pm 0.01$ & $0.55 \pm 0.03$ & $0.63 \pm 0.04$ & $0.31 \pm 0.06$ \\
\hline 6 & $00^{\mathrm{h}} 42^{\mathrm{m}} 46^{\mathrm{s}} .3$ & $41^{\circ} 08^{\prime} 36^{\prime \prime}$ & 1.3 & -5.0 & 5.1 & 91 & $0.096 \pm 0.008$ & $0.22 \pm 0.02$ & $0.28 \pm 0.03$ & $0.12 \pm 0.01$ \\
\hline 7 & $00^{\mathrm{h}} 42^{\mathrm{m}} 11^{\mathrm{s}} .2$ & $41^{\circ} 07^{\prime} 06^{\prime \prime}$ & 2.5 & -0.6 & 2.6 & 200 & $0.57 \pm 0.02$ & $1.5 \pm 0.1$ & $2.4 \pm 0.1$ & $1.4 \pm 0.1$ \\
\hline 8 & $00^{\mathrm{h}} 42^{\mathrm{m}} 29^{\mathrm{s}} .3$ & $41^{\circ} 18^{\prime} 14^{\prime \prime}$ & 0.0 & 3.6 & 3.6 & 110 & $0.26 \pm 0.02$ & $0.71 \pm 0.04$ & $1.2 \pm 0.1$ & $0.78 \pm 0.14$ \\
\hline 9 & $00^{\mathrm{h}} 42^{\mathrm{m}} 21^{\mathrm{s}} .5$ & $41^{\circ} 18^{\prime} 26^{\prime \prime}$ & 0.2 & 4.9 & 4.9 & 130 & $0.13 \pm 0.01$ & $0.34 \pm 0.03$ & $0.45 \pm 0.10$ & $<0.40$ \\
\hline 10 & $00^{\mathrm{h}} 42^{\mathrm{m}} 34^{\mathrm{s}} .7$ & $41^{\circ} 21^{\prime} 23^{\prime \prime}$ & -0.7 & 4.7 & 4.8 & 140 & $1.2 \pm 0.1$ & $2.6 \pm 0.1$ & $3.5 \pm 0.3$ & $1.3 \pm 0.3$ \\
\hline
\end{tabular}

(This table is available in its entirety in machine-readable form.)

SPIRE (Bendo 2011) and PACS (Muller et al. 2011) factors in an iterative loop. The median correction at each wavelength was $<2 \%$. Most of the regions extracted by CSAR are larger than the telescope PSF (see Figure 5), so we use the SPIRE extended source calibration.
Herschel color-corrected fluxes at 350, 250, 160, and $100 \mu \mathrm{m}$ for each cloud are listed in Columns 8-11 of Table 1. A $500 \mu \mathrm{m}$ flux is not listed as these data have a resolution lower than that of the extraction wavelength. One-sigma errors are listed for each cloud; these are the statistical errors based on the local pixel 


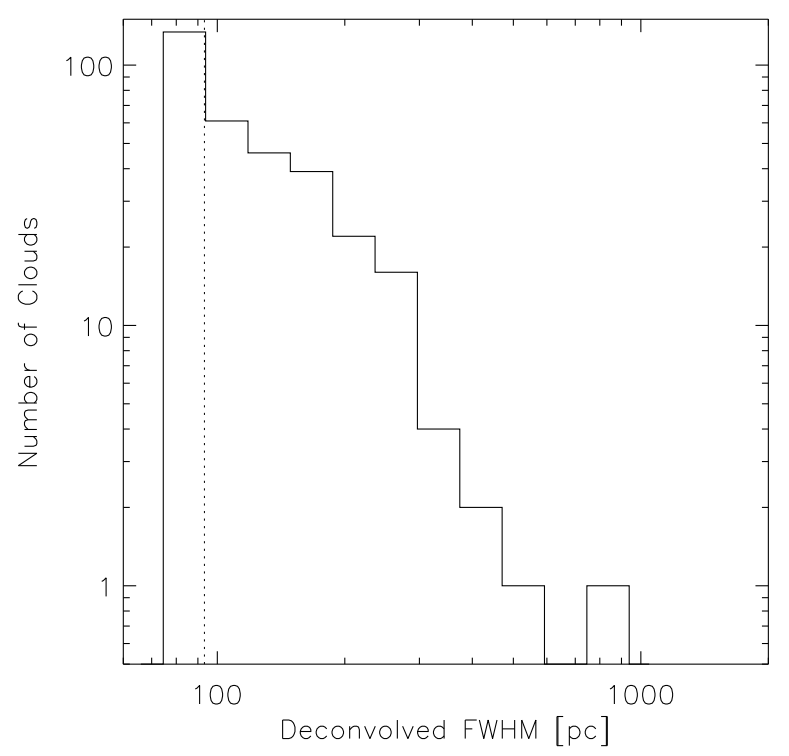

Figure 5. Histogram of deconvolved cloud FWHMs. The vertical dashed line shows the beam FWHM.

rms and do not include the systematic calibration uncertainties. Upper limits are given for clouds whose measured flux was less than $3 \sigma$. Given that the majority of the clouds are extended, the calibration error is $12 \%$ for the SPIRE bands (Valtchanov 2011) and $10 \%$ for the PACS (Paladini et al. 2012).

\section{MOLECULAR CLOUD PROPERTIES}

\subsection{Cloud Size}

For each cloud, a major and minor FWHM is calculated from the moments of the half-power contour. The geometric mean of the deconvolved major and minor FWHMs is then taken as the cloud's projected size as listed in Column 7 of Table 1 . The histogram of these sizes is shown in Figure 5. The vertical dotted line shows the $350 \mu \mathrm{m}$ beam FWHM; this is equivalent to $93 \mathrm{pc}$ at the assumed distance to M31. The bin to the left of this line is caused by the statistical scatter in the FWHM estimates for unresolved clouds (all data points inside it have a deconvolved FWHM that is within 0.5 pixels of the resolution limit). It is assumed that a cloud's cross section is not affected by the projection of M31.

The histogram shows a range of cloud sizes starting with unresolved sources. The majority of the clouds have sizes within 5 times the beam FWHM ( $\sim 500 \mathrm{pc})$. There is one source that is larger than $700 \mathrm{pc}$; this is HELGA 319 located at $X=-19.4$, $Y=6.3$, which is a large, low surface brightness feature. For comparison to these sizes, one of the nearest galactic GMCs, the Taurus molecular cloud, has a diameter of $\sim 25 \mathrm{pc}$, and the Gould Belt, the local system of GMCs, has a diameter of $1 \mathrm{kpc}$. Additionally, the mean size of a GMC in the Milky Way is $\sim 40 \mathrm{pc}$ (Solomon et al. 1979) and in the LMC is $\sim 30 \mathrm{pc}$ (Hughes et al. 2010). Thus, given this size distribution, the clouds we are extracting are probably complexes of GMCs and not the equivalent of individual GMCs. Comparison of cloud boundaries with published interferometry results (see Section 4.5) shows that these sources are indeed substructured below our resolution limits.

Figure 6 shows the number density of clouds (FIR sources) per square kiloparsec as a function of galactocentric distance. Error bars are shown assuming normal errors. There is a

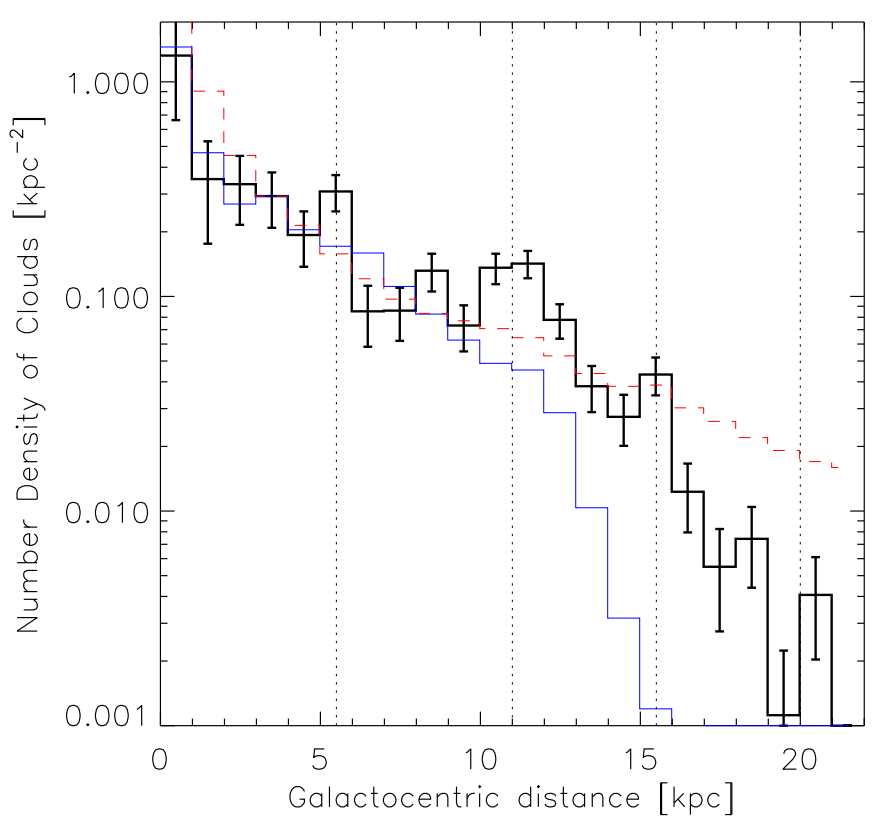

Figure 6. Number density of clouds with galactocentric distance. The vertical dashed line shows the location of peaks coincident with ring structures. The solid blue curve shows the number density of dark nebulae from Hodge (1980), and the red dashed curve shows the surface brightness profile of M31 at $3.6 \mu \mathrm{m}$. Both the red and blue curves have been normalized against the black curve at $3-4 \mathrm{kpc}$.

clear downward trend out to $20 \mathrm{kpc}$; this is the region where the contiguous $250 \mu \mathrm{m}$ emission from Andromeda-and thus the single-structure tree associated with it-blends into the background. The fraction of optical light from the disk has also dropped off significantly at this radius (Courteau et al. 2011), although the disk features can be detected out to $40-50 \mathrm{kpc}$ (Ibata et al. 2005; Courteau et al. 2011; Fritz et al. 2012). While there is scatter in this plot, it does show a series of peaks at $\sim 5 \mathrm{kpc}$ intervals (i.e., 5,11 , and $15 \mathrm{kpc}$ as shown by the dotted vertical lines) coincident with the observed rings at those distances. It is interesting to note that these follow the same pattern $(\sim 20,25$, and $30 \mathrm{kpc})$ of FIR features in M31's outermost regions as detected by Paper I. These features could be a system of weak resonant rings (e.g., Jungwiert \& Palous 1996; Buta 1999).

Also shown in Figure 6 is the number density of dark nebulae (solid blue curve) from Hodge (1980) and the surface brightness profile of M31 at $3.6 \mu \mathrm{m}$ (dashed red curve). Both the dark nebulae and $3.6 \mu \mathrm{m}$ profiles have been normalized against the distribution of FIR clouds at a radius of 3-4 kpc. Both curves broadly follow the distribution of FIR clouds out to a radius of $\sim 8 \mathrm{kpc}$, with the exception of the peak at $5 \mathrm{kpc}$. The $10 \mathrm{kpc}$ feature is seen as a significant enhancement above the $3.5 \mu \mathrm{m}$ profile, but it is not seen at all in the profile of dark nebulae. The distribution of dark nebulae drops off quite dramatically beyond $10 \mathrm{kpc}$. It is possible that this is a selection effect; the distribution of the dark nebulae is correlated with the surface brightness of the disk because it is that which determines the background contrast and thus the chance of detecting a dark nebula (Jackson et al. 2008). Thus, it is possible that the optical surface brightness of M31 beyond $10 \mathrm{kpc}$ may not have provided sufficient contrast to discern nebulae against in the Hodge (1980) survey.

The peak of the size distribution of the Hodge (1980) dark nebulae is at $\sim 100-150 \mathrm{pc}$, not dissimilar to our resolution 
limit. A comparison of positions between the Hodge (1980) dark nebulae and our FIR-selected clouds shows that only about $5.8 \%$ of FIR cloud positions are within $100 \mathrm{pc}$ of a dark nebulae reference position. This only increases to $17 \%$ if the search separation is increased to $200 \mathrm{pc}$. The large-scale distribution of FIR emission in galaxies does correlate with gas column density (e.g., Neininger et al. 1996), so this discrepancy between features in the FIR data and the position of dark nebulae is interesting. Hodge (1980) himself noted the poor correlation between the distribution of dark nebulae and the distribution of atomic hydrogen. It is possible that dark nebulae poorly match the FIR clouds because they are just surface features seen against the bright disk of M31 (see the $3.5 \mu \mathrm{m}$ profile), whereas the FIR clouds trace emission through the entire depth of the disk. A similar feature is seen in the distribution of infrared dark clouds (IRDCs) in the plane of the Milky Way (Jackson et al. 2008). It is possible therefore that analyzing dark features (dark nebulae) in a disk may not give a true representation of the distribution of clouds in that galaxy (Wilcock et al. 2012).

\subsection{Cloud Mass}

\subsubsection{Mass Calculation}

For each cloud that has a flux measurement with $\mathrm{S} / \mathrm{N}>3 \sigma$ at three or more wavelengths between 100 and $350 \mu \mathrm{m}$, we follow Paper II and fit a modified-blackbody function of the form

$$
S_{v}=\frac{B_{v}\left(T_{d}\right) \kappa_{v} M_{d}}{D^{2}}
$$

where $S_{v}$ is the flux density at frequency $v, B_{v}\left(T_{d}\right)$ is the Planck function for a blackbody with temperature $T_{d}, M_{d}$ is the dust mass, and $D$ is the distance to the source. The dust absorption coefficient, $\kappa_{\nu}$, was parameterized as a power law with the form $\kappa_{v} \propto \nu^{\beta}$, where $\beta$ is the dust emissivity index. The dust absorption coefficient was scaled from a reference value of $0.192 \mathrm{~cm}^{2} \mathrm{~g}^{-1}$ at $350 \mu \mathrm{m}$ (Draine 2003). This value is the same as used for Paper I and Paper II. The uncertainty in $\kappa_{v}$ could be as large as a few and is ignored when quoting uncertainties on the mass estimates. The value of $\beta$ for each cloud was estimated using the cloud's galactocentric distance and the radial dust emissivity relationship from Paper II.

We convert the dust mass, $M_{d}$, into a total mass (i.e., gas and dust), $M_{\text {cloud }}$, taking into account the metallicity gradient in M31 by using the radial dust-to-gas relationship from Paper II. There are therefore only two free parameters, $T$ and $M_{\text {cloud }}$, for each fit. The Levenberg-Marquardt least-squares minimization package MPFIT (Markwardt 2009) was used for all fitting, and the fitting was done in $\log v$ versus $\log v F_{v}$ parameter space. Hereafter, quoted cloud masses refer to the total mass of dust and gas unless stated otherwise.

The fitted masses and temperatures are listed in Columns 2 and 3 , respectively, of Table 2 . The clouds have a wide range of masses from $2.5 \times 10^{4}$ to $1.4 \times 10^{7} M_{\odot}$, with a median mass of $4.1 \times 10^{5} M_{\odot}$. In Paper I the total of molecular gas in M31 was estimated to be $2.6 \times 10^{8} M_{\odot}$. The total mass of all leaf-node sources for which there is a valid SED fit is $1.6 \times 10^{8} M_{\odot}$. The equivalent total mass for all leaf nodes in the $10 \mathrm{kpc}$ branch of the dendrogram is $6.5 \times 10^{7} M_{\odot}$. Extracted clouds in the $10 \mathrm{kpc}$ ring thus account for $25 \%$ of the molecular gas in M31.

\subsubsection{Mass Completeness}

Figure 7 shows the normal $N\left(M_{\text {cloud }}\right)$ and cumulative $N\left(>M_{\text {cloud }}\right)$ mass distributions for the clouds in M31. Assess-
Table 2

Table of SED Parameters

\begin{tabular}{lcccc}
\hline \hline $\begin{array}{l}\text { Name } \\
\text { (HELGA) }\end{array}$ & $\begin{array}{c}M_{\text {cloud }} \\
\left(10^{5} M_{\odot}\right)\end{array}$ & $\begin{array}{c}T \\
(\mathrm{~K})\end{array}$ & $\begin{array}{c}L_{\mathrm{FIR}} \\
\left(10^{5} L_{\odot}\right)\end{array}$ & $\begin{array}{c}L_{\mathrm{CO}} \\
\left(10^{4} \mathrm{~K} \mathrm{~km} \mathrm{~s}^{-1} \mathrm{pc}^{2}\right)\end{array}$ \\
\hline 1 & 8.7 & 23 & 15. & $\cdots$ \\
2 & 2.9 & 21 & 4.0 & $\cdots$ \\
3 & 2.4 & 16 & 4.2 & 2.2 \\
4 & 1.9 & 16 & 4.1 & 3.4 \\
5 & 1.9 & 16 & 2.7 & 6.7 \\
6 & 0.72 & 16 & 1.1 & $<0.59$ \\
7 & 2.6 & 18 & 10. & 2.4 \\
8 & 1.2 & 18 & 5.3 & $<3.8$ \\
9 & 0.78 & 18 & 2.1 & $<2.7$ \\
10 & 8.9 & 16 & 13. & 22. \\
\hline
\end{tabular}

(This table is available in its entirety in machine-readable form.)

ing the completeness of the mass distribution of the extracted sources is complicated by the radial gradients in the properties of the dust and the nonuniform distribution of sources relative to those gradients. This is best illustrated by our point-source mass sensitivity, which is shown by the dotted curve plotted over the mass distribution. It was calculated by 100,000 repetitions of the mass calculation from our limiting flux sensitivity of $S_{250 \mu \mathrm{m}}=54 \mathrm{mJy}$ (a $5 \sigma$ point source at $250 \mu \mathrm{m}$; see the CSAR extraction criteria), where the gas-to-dust ratio and the dust temperature have been sampled from normal distributions with the same mean and standard deviation as the sources in our catalog $\left(r_{\mathrm{gd}}=74 \pm 36, T=18 \pm 2 \mathrm{~K}\right)$. The resulting point-source sensitivity is $6.6 \pm 4.5 \times 10^{5} M_{\odot}$. This corresponds to the lower tail of the mass distribution, but it does not account for the deviation of the distribution from a power law between $10^{5}$ and $10^{6} \mathrm{M}_{\odot}$.

To simplify the assessment of our completeness, we only consider point sources (the highest bin in the size histogram). Additionally, we consider two extremes of background-a "high" $45^{\prime} \times 12^{\prime}$ background running along the southeast portion of the $10 \mathrm{kpc}$ ring and a "low" background of the same size immediately to the southeast of it. We replicated the source extraction process on these backgrounds after injecting $250 \mu \mathrm{m}$ point sources into the field with masses drawn from the fitted power-law distribution (see below) and with the same dust properties as those used in the previous test. A total of 10,000 sources were injected onto each background in batches of 25 . It was found that the mass distributions of synthetic point sources were $50 \%$ complete above a mass of $1.6 \times 10^{5} M_{\odot}$ for the low and $4.5 \times 10^{5} M_{\odot}$ for the high backgrounds, respectively. These limits are plotted on the mass distribution in Figure 7. Therefore, the departure of the mass distribution away from the power-law rise below $10^{5} M_{\odot}$ is due to incompleteness.

\subsubsection{Power-law Mass Distributions}

The higher-mass portion of the binned mass distribution (Figure 7 left hand panel) can be fit by a power law of the form $N(M) \propto M^{-\alpha_{M}}$. For the Andromeda clouds, least-squares fitting to the power law above $4.5 \times 10^{5} M_{\odot}$ gives a best-fit exponent of $\alpha_{M}=1.21 \pm 0.23$ (shown by the dot-dashed line). However, these power laws can be easily biased by small number statistics in the higher-mass bins (e.g., Maschberger \& Kroupa 2009). A more reliable way to display the data is as a cumulative mass distribution. The cumulative mass function for GMCs in a selection of nearby galaxies consists of a linear tail below $\sim 10^{5} M_{\odot}$ that steepens at higher masses (Blitz et al. 2007; Fukui \& Kawamura 2010). The cumulative mass distribution of M31's clouds is shown by the right-hand plot in Figure 7. It 

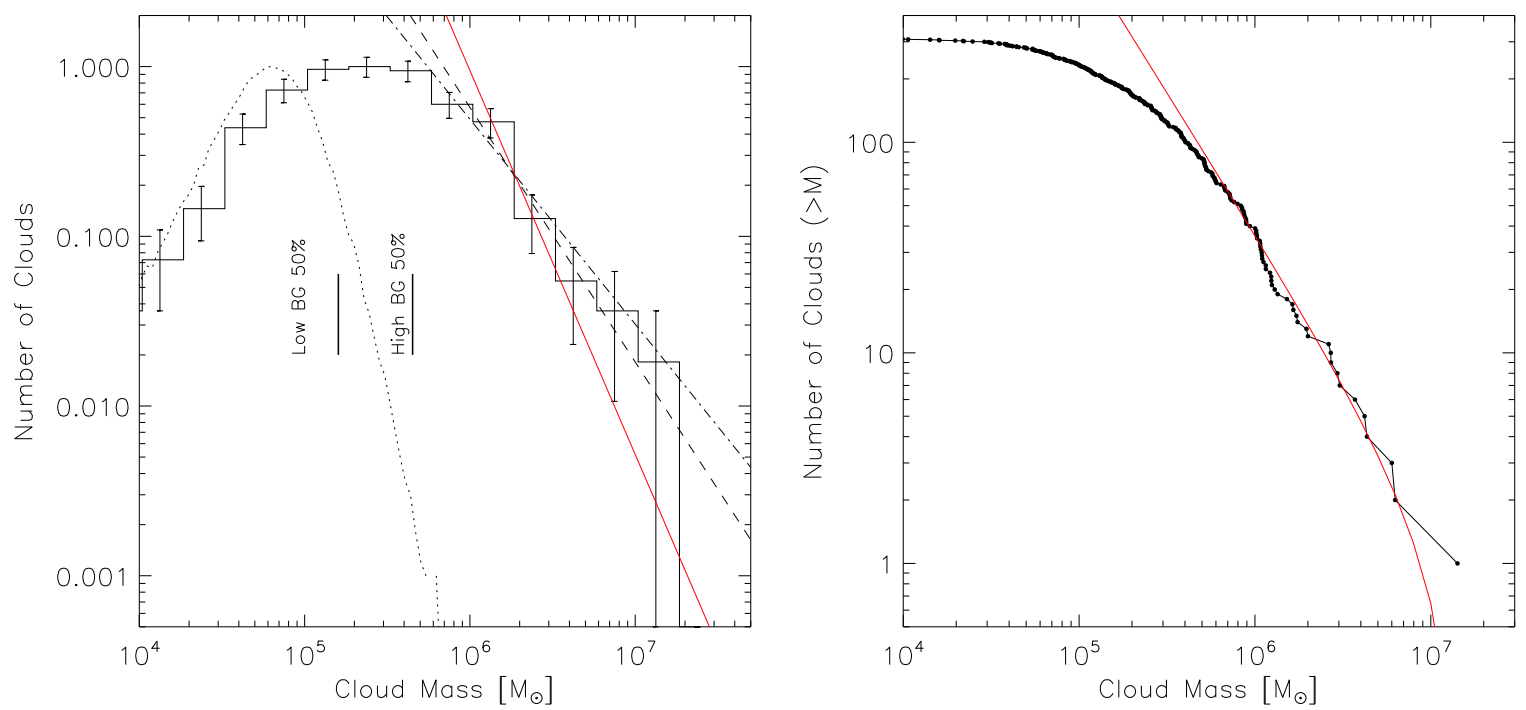

Figure 7. Mass distribution of clouds in M31. The solid red curves in both plots show mass distributions calculated from a truncated power law with an exponent $\alpha_{M}=2.34 \pm 0.12$ (equivalent to an exponent of 2.34-1 in the cumulative case; see text for more details). Left: histogram of $M_{\text {cloud }}$. The dot-dashed power law has an exponent of 1.21 and is a least-squares fit to the normal histogram. This is significantly different than that found via the MML method (i.e., 2.34). The dashed power law shows the equivalent $\alpha=1.5$ power law for Milky Way clouds. The bars along the bottom edge show the 50\% point-source completeness for low and highly structured background (see the text for explanation). The dotted line shows our equivalent point-source sensitivity once variations of dust temperature and properties have been accounted for. Right: cumulative histogram of total cloud mass for the M31 clouds.

is also flat below $10^{5} M_{\odot}$ and then begins to turn over between $10^{5}$ and $10^{6} M_{\odot}$.

For an infinite mass distribution of the form $N(M) \propto$ $M^{-\alpha_{M}}$, the cumulative distribution is given by $N(>M) \propto$ $M^{-\left(\alpha_{M}-1\right)}$. Following Gratier et al. (2012), we use the modified maximum likelihood (MML) estimator given by Maschberger \& Kroupa (2009) to estimate the exponent of the underlying mass distribution shown in Figure 7. The MML estimator for $\alpha_{M}$ has a value of $2.34 \pm 0.12$ for the Andromeda clouds. The error on the estimate is found using the same bootstrap method as used by Gratier et al. (2012). The lower truncation limit was again taken as $4 \times 10^{5} M_{\odot}$. The estimate for the upper truncation limit is $1.4 \times 10^{7} M_{\odot}$.

The mass distributions calculated from $\alpha=2.34$ are shown by the solid red curves in Figure 7 . For clouds just in the $10 \mathrm{kpc}$ ring branch the estimate is $\alpha_{M}=2.62 \pm 0.21$, which is consistent with the estimate for the set of all clouds. Low number statistics meant that it was not possible to reliably estimate a value of $\alpha_{M}$ for just clouds interior or exterior to the ring. The least-squares and MML estimates appear to follow opposite sides of the error bars of the binned data.

Molecular clouds in the Milky Way have an exponent of $\alpha_{M} \sim 1.5$ (e.g., Sanders et al. 1985; Solomon et al. 1987; RomanDuval et al. 2010); this is shown by the dashed black line in Figure 7 for comparison. The Milky Way exponent is within $3 \sigma$ of the Andromeda exponent and passes through most of the error bars above $10^{6} M_{\odot}$. Our estimate of $\alpha_{M}$ is consistent with a value of $\left(\alpha_{M}-1\right)=1.55 \pm 0.2$ found from high-resolution CO mapping of a subset of M31 GMCs mapped with the BIMA interferometer (R07, Blitz et al. 2007). It is also consistent with the value of $2.2 \pm 0.3$ found for GMCs in M33 using the same technique (Gratier et al. 2012).

\subsection{Temperature and Luminosity}

\subsubsection{FIR Luminosity Function}

A histogram of temperatures obtained from the SED fitting is shown in the left panel of Figure 8. The majority of cloud dust temperatures are in the range $15-25 \mathrm{~K}$, with a median value of $18 \mathrm{~K}$. This is not unexpected as we are fitting a single-temperature component over the wavelength range $100-350 \mu \mathrm{m}$ and are therefore going to be dominated by the cold dust component. We calculate the luminosity, $L_{\mathrm{FIR}}$, of this component by integrating beneath the fitted SED gray body in the range $10-1000 \mu \mathrm{m}$. The individual values of $L_{\mathrm{FIR}}$ are listed in Column 4 of Table 2.

The cumulative luminosity function is shown in the righthand panel of Figure 8. Following the MML procedure used on the mass distribution (see above), the MML estimate for the power-law exponent of the luminosity function $\left(N(L) \propto L^{-\alpha_{M}}\right)$ was found to be $\alpha_{L}=2.13 \pm 0.15$. This is similar to the FIR luminosity function for clouds in the Milky Way found by Harris \& Clegg (1983) and to the CO luminosity function of clouds in M33 (Gratier et al. 2012; Rosolowsky et al. 2007). It is also very similar to the exponent found for the mass distribution above.

\subsubsection{Star Formation Rate Indicators}

Various continuum and multiwavelength products have been used to calculate SFRs (see Kennicutt 1998a; Kennicutt \& Evans 2012 for reviews), with many of these tracing their reasoning back to the Kennicutt-Schmidt law (Schmidt 1959; Kennicutt 1998b). This law assumes that the rate of star formation is proportional to some power of the interstellar gas surface density. Thus, a measurement that determines the gas density or mass can be used as a proxy for the SFR. Two of these measures are the infrared luminosity and the mid-infrared flux density. Figure 9 shows a plot of $L_{\mathrm{FIR}}$ with Spitzer $24 \mu \mathrm{m}$ flux density for the M31 clouds. The Spitzer values were measured in the same manner as for the Herschel fluxes and used the same source masks. About $11 \%$ of the sources for which we have $L_{\mathrm{FIR}}$ estimates are undetected at $24 \mu \mathrm{m}$, and this ratio appears constant with galactocentric distance.

Figure 9 shows that the $24 \mu \mathrm{m}$ flux density, which is a tracer of warm dust and thus a tracer for the amount of ongoing star formation, correlates on a cloud/complex scale with the 

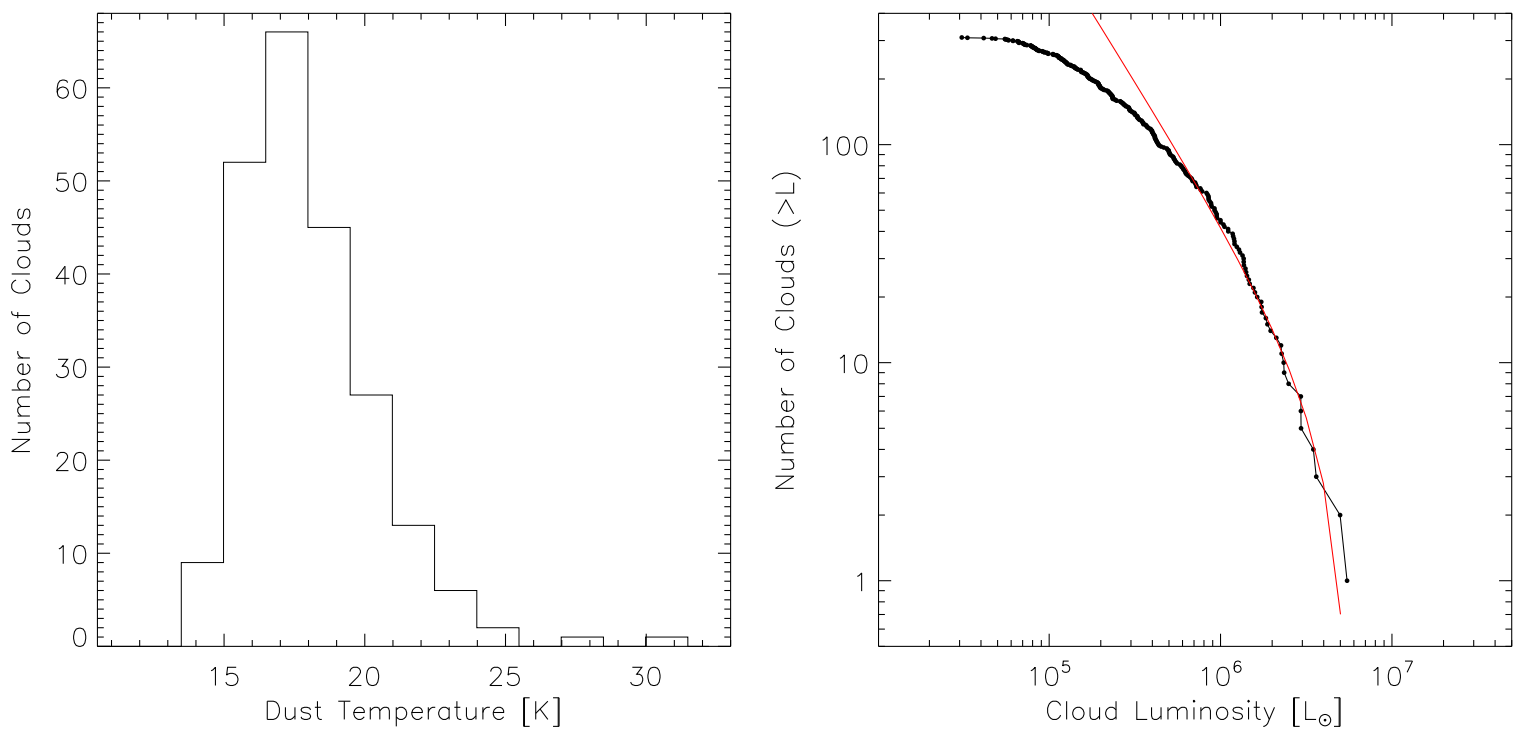

Figure 8. Temperature and luminosity of M31 clouds. Left: Histogram of dust temperature fitted between the wavelengths of 100 and $350 \mu \mathrm{m}$. Right: Cumulative histogram of FIR luminosity $L_{\text {FIR }}$ integrated beneath the best-fit SED gray body. The red line shows the luminosity function calculated from a power law with an exponent of $\alpha_{L}=2.1 \pm 0.3$.

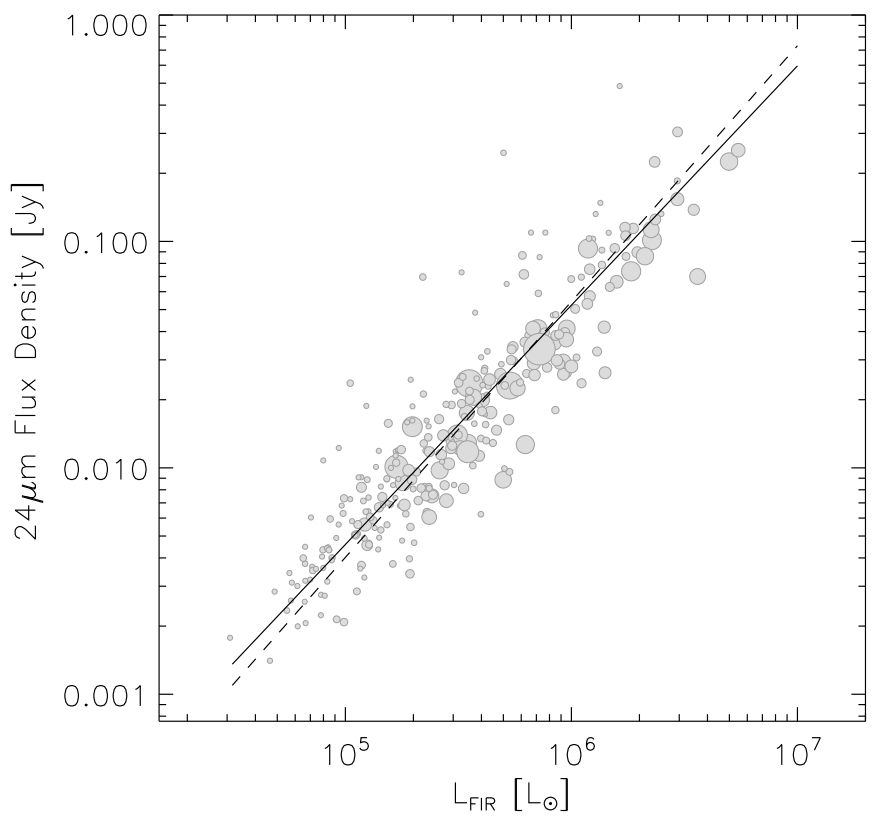

Figure 9. Plot showing the correlation between FIR luminosity and mid-infrared flux density. The width of the symbols shows the relative radius of the clouds. The solid line is a line of best fit; the correlation coefficient is 0.9 . The dashed line indicates the slope of the relationship from Vutisalchavakul \& Evans (2013).

luminosity of cool dust, which traces the reservoir of gas available at the start of the star formation process. Following Vutisalchavakul \& Evans (2013), the SFR calculated from the FIR continuum is $\mathrm{SFR}_{\mathrm{FIR}} \propto L_{\mathrm{FIR}}$ (Kennicutt 1998a), while the SFR calculated from $S_{24 \mu \mathrm{m}}$ is $\mathrm{SFR}_{24 \mu \mathrm{m}} \propto S_{24 \mu \mathrm{m}}^{0.88}$ (Calzetti et al. 2007). Eliminating the SFR between these gives $S_{24 \mu \mathrm{m}} \propto L_{\mathrm{FIR}}^{1.13}$. This power law is shown as the dashed line in Figure 9.

A best fit to the data gives

$\log \left(S_{24 \mu \mathrm{m}}\right)=(1.057 \pm 0.003) \log \left(L_{\mathrm{FIR}}\right)-\log \left(2.4 \pm 0.1 \times 10^{-8}\right)$,

where $S_{24 \mu \mathrm{m}}$ is in Jy and $L_{\mathrm{FIR}}$ is in $L_{\odot}$. The best fit is shown by the solid line. The exponents of the relationship and best fit are approximately equal. The correlation coefficient for this distribution is 0.90. Vutisalchavakul \& Evans (2013) showed that the relationship also holds observationally for low-mass star formation regions within $1 \mathrm{kpc}$ of the Sun and for highermass star formation regions scattered throughout the Milky Way. The theoretical relationship appears to be consistent with the Andromeda data. This further reinforces the idea that the properties of the clouds in M31 are consistent with the expected properties of clouds in the Milky Way. The full SFR for these clouds and a detailed comparison with the results of Paper III will be discussed in a follow-up paper.

\subsection{CO Luminosity}

The usual molecule for tracing giant molecular gas clouds is carbon monoxide, but there have been few comprehensive surveys across the entire disk of M31. Nieten et al. (2006) produced the first complete, subarcminute resolution CO study of M31, but there has not, as yet, been a published catalog of individual $\mathrm{CO}$ clouds. We therefore use our catalog to measure CO luminosities from the Nieten et al. CO map.

Nieten et al. (2006) mapped M31 in the $J=1-0$ line of ${ }^{12} \mathrm{CO}$ with a resolution of $23^{\prime \prime}$ using the IRAM $30 \mathrm{~m}$ telescope. This resolution is nearly equal to that of Herschel at $350 \mu \mathrm{m}$, our working resolution, making comparison of our data with the Nieten CO relatively straightforward. Figure 10 shows a comparison of the ${ }^{12} \mathrm{CO}$ emission (top panel) and the $250 \mu \mathrm{m}$ emission (middle panel) toward Andromeda. The box outline shows the limit of the $\mathrm{CO}$ data. A single $10 \sigma{ }^{12} \mathrm{CO}$ contour is shown in both images, where $\sigma=0.35 \mathrm{~K} \mathrm{~km} \mathrm{~s}^{-1}$ (Nieten et al. 2006). The CO intensity traces well the peaks of the dust continuum. However, the extended dust component is not detected in the $\mathrm{CO}$, showing that the $\mathrm{CO}$ emission is confined only to the densest regions.

That dust emission traces CO luminosity well has been shown for a sample of galaxies, including M31 (Eales et al. 2012); we can now test whether this holds for clouds inside M31 using the CSAR extraction contours to measure an integrated $\mathrm{CO}$ luminosity $L_{\mathrm{CO}}$ for each Herschel cloud using the Nieten CO map. CO luminosities in the range $10^{4}-10^{6} \mathrm{~K} \mathrm{~km} \mathrm{~s}^{-1} \mathrm{pc}^{-2}$ were measured. The bottom panel of 10 shows the location of the 


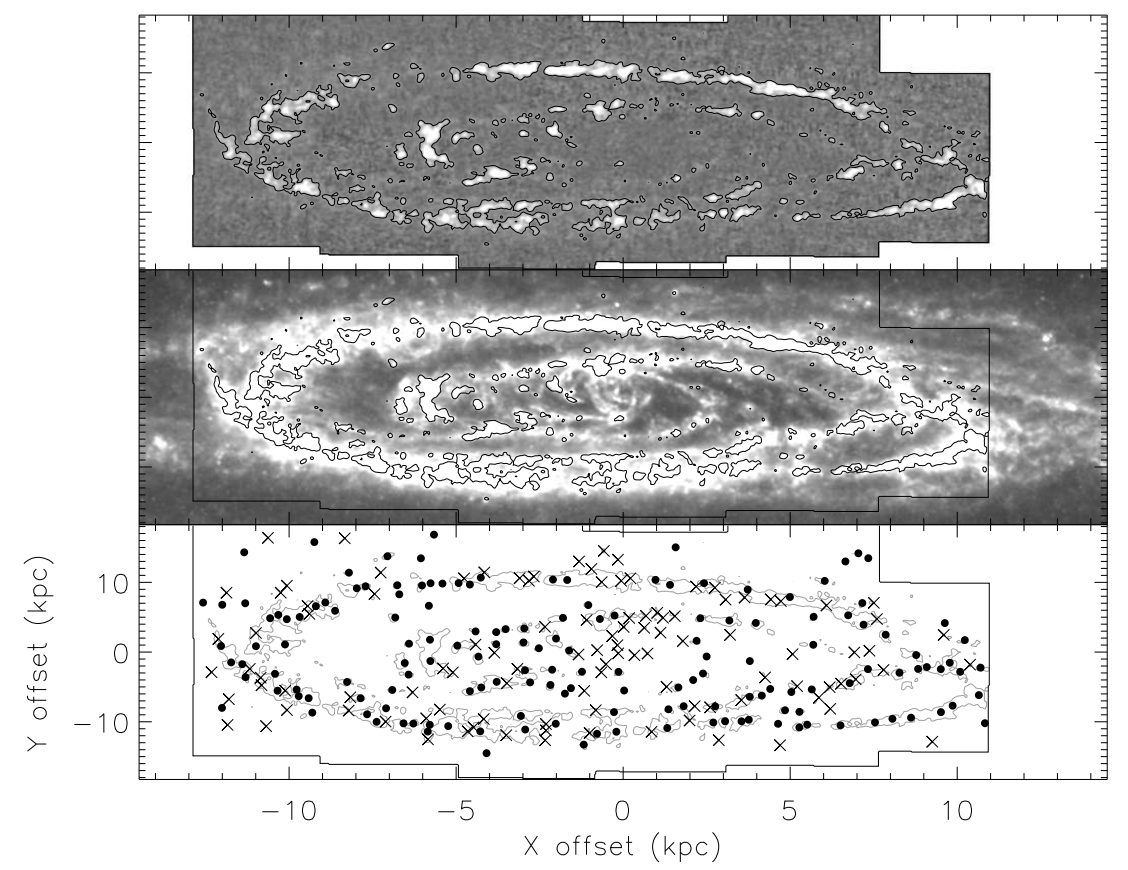

Figure 10. Comparison of (top) IRAM ${ }^{12} \mathrm{CO}$ integrated emission (Nieten et al. 2006) and (middle) SPIRE $250 \mu \mathrm{m}$ dust emission toward the Andromeda galaxy. The bottom panel shows which of the Herschel GMCs were detected (filled circle) or not detected (crosses) in $\mathrm{CO}$ emission. The same $10 \sigma^{12} \mathrm{CO}$ contour is plotted over each map.

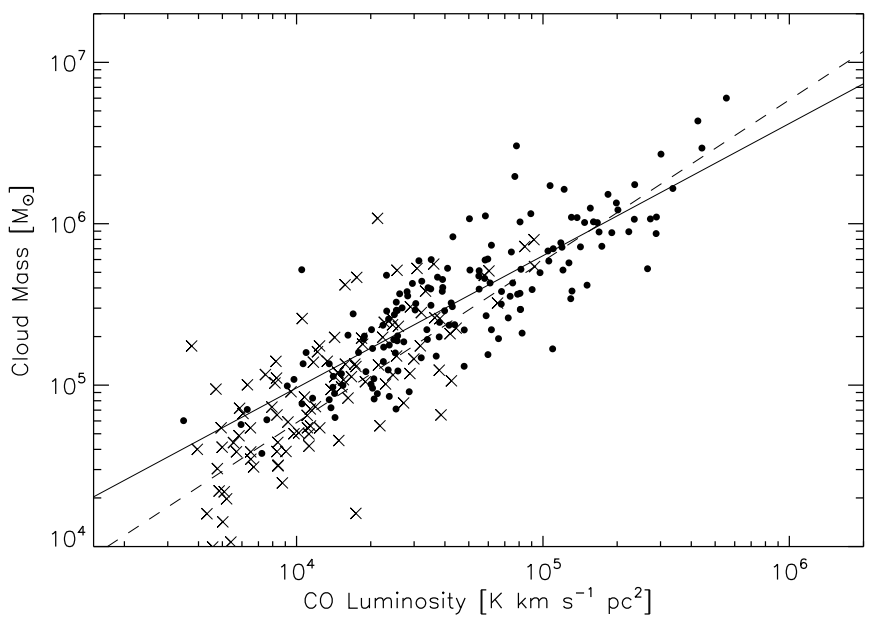

Figure 11. Cloud ${ }^{12} \mathrm{CO}$ luminosity vs. total mass derived from the FIR. The solid line shows a best-fit power law with an exponent of $0.82 \pm 0.05$. The dashed line shows the $\alpha_{\mathrm{CO}}$ relationship from Paper II.

Herschel sources that were detected at greater than $3 \sigma$ in the $\mathrm{CO}$ data (filled circles) and those that were not detected (crosses).

Figure 11 shows $L_{\mathrm{CO}}$ versus $M_{\text {cloud }}$. The black dots show clouds that have a $\mathrm{CO}$ detection, while the crosses show $3 \sigma$ $\mathrm{CO}$ upper limits for undetected clouds. There is a clear trend between the mass and $\mathrm{CO}$ luminosity. We test the correlation by performing a linear regression to the $\mathrm{CO}$ detections. This gives a best fit of

$$
\log \left(M_{\text {cloud }}\right)=(0.82 \pm 0.04) \log \left(L_{\mathrm{CO}}\right)+(1.7 \pm 0.2)
$$

where $M_{\text {cloud }}$ is in $M_{\odot}$ and $L_{\mathrm{CO}}$ is in $\mathrm{K} \mathrm{km} \mathrm{s}^{-1} \mathrm{pc}^{2}$. This is shown by the solid line. The correlation coefficient for this fit is 0.84 , showing a reasonable correlation between the mass of a cloud derived from the Herschel data $\left(M_{\text {cloud }}\right)$ and that cloud's CO luminosity. The $\mathrm{CO}$ upper limits appear to broadly follow the same trend, at least as far as low $L_{C O}$ correlates with low $M_{\text {cloud }}$.
The dashed line in Figure 11 shows the Paper II relation of $\alpha_{\mathrm{CO}}=M_{\text {cloud }}\left(H_{2}\right) / L_{\mathrm{CO}}=4.1 M_{\odot} \mathrm{pc}^{-2} \mathrm{~K}^{-1} \mathrm{~km}^{-1} \mathrm{~s}$ under the assumption that $M_{\text {cloud }}$ is $70 \% \mathrm{H}_{2}$ by mass (protosolar abundance; e.g., Asplund et al. 2009).

Solomon et al. (1987) presented a CO survey of clouds in the Milky Way observed with the $14 \mathrm{~m}$ FCRAO antenna. The implied sizes for their clouds are lower than we have measured for M31, but the range of $\mathrm{CO}$ luminosities is almost identical to those we calculate. This supports the proposition that we are not resolving individual clouds and are instead resolving assemblages of individual clouds. The upper range for both surveys is $L_{\mathrm{CO}}=\sim 10^{6} \mathrm{~K} \mathrm{~km} \mathrm{~s}^{-1} \mathrm{pc}^{-2}$. They find a best fit between the virial mass $M_{\text {virial }}$ of each cloud and $L_{\mathrm{CO}}$ of

$$
\log \left(M_{\text {virial }}\right)=0.81 \log \left(L_{\mathrm{CO}}\right)+1.6 .
$$

This is virtually identical to the power law that we fit to the clouds in Andromeda.

Solomon et al. related the optically thick ${ }^{12} \mathrm{CO}$ luminosity, which is proportional to the cloud's cross section, to the virial mass using a size-line width relation to give $M_{\text {virial }}=$ $43 L_{\mathrm{CO}}^{4 / 5} M_{\odot}$. This relationship would hold for virialized clouds. That we obtain the same mass-luminosity relationship in M31 suggests that these clouds are virialized on some level. This would probably not be on the complex scale, but it could be at some spatial scale below our resolution limit (i.e., the complexes are made up of unresolved virialized units).

\subsection{Comparison with Interferometry Studies}

Studies of individual GMCs in M31 with interferometers have been made (Vogel et al. 1987; Wilson \& Rudolph 1993; Allen et al. 1995; Loinard \& Allen 1998); the increasing sensitivity of millimeter interferometers has meant that studies of more than a few clouds at a time are now possible (R07; S08). R07 mapped a $\sim 7 \mathrm{kpc}$ arc along a northwestern section of the $10 \mathrm{kpc}$ ring (among other fields). They detected 19 clouds for which 

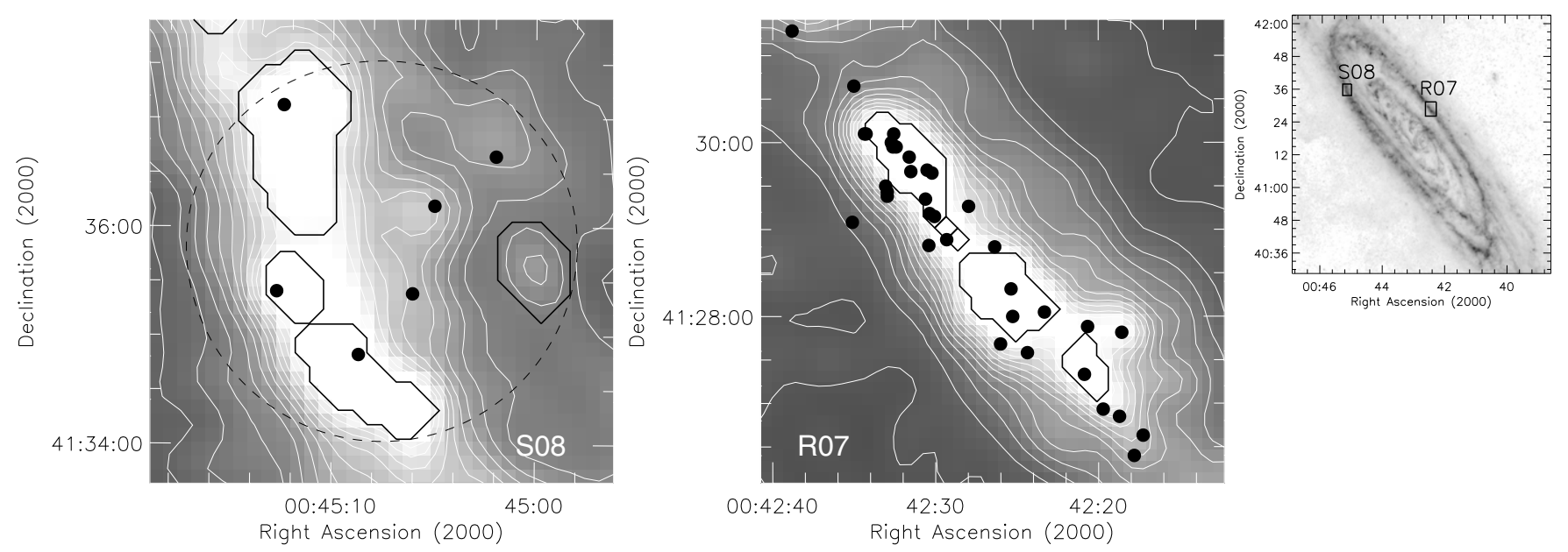

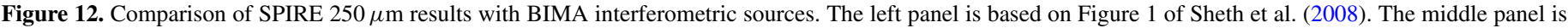

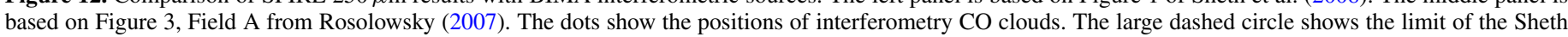

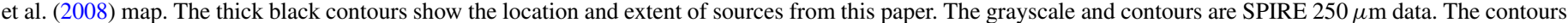
are spaced at $5 \sigma$ intervals. The smaller right-hand panel shows the location of the other two panels.

they could accurately resolve the properties and a further 48 unresolved clouds. S08 mapped a single $2^{\prime}$ diameter field across a northeastern section of the $10 \mathrm{kpc}$ ring, detecting six clouds.

Figure 12 shows a comparison of the results from these two studies using the BIMA interferometer and the Herschel data presented in this paper. The grayscale and contours are SPIRE $250 \mu \mathrm{m}$ dust emission with contour spacings of $5 \sigma$. The middle panel shows the region coincident with the R07 Field A, while the left-hand panel shows the S08 field. The BIMA clouds are shown by the markers (only the resolved sources are shown for the R07 field). R07 and S08 have resolutions that are $\sim 3$ times the resolution of the $350 \mu \mathrm{m}$ Herschel maps. Comparison with the R07 field shows that the leaf nodes identified in this paper can break into multiple objects when viewed with an interferometer. Typically there is one BIMA source identified with the Herschel peak and several more sources clustered around it. The S08 field shows a similar pattern. They did not break down their clouds into subfragments as R07 did, but the detailed structure is still visible in their original maps.

The comparison of the BIMA data to the Herschel regions shows that it is correct to think of the Herschel regions as complexes of GMCs and not individual clouds. This comparison also illustrates the difficulty of comprehensively mapping a source as large as M31 with sufficient resolution to resolve individual star formation regions. The speed and sensitivity of ALMA would go a long way to solving this, but its relatively high declination and large size make observing M31 challenging _ if not virtually impossible-from ALMA's location. It would, however, make an excellent target for the proposed NOEMA array at IRAM.

\section{GLOBAL STRUCTURE}

\subsection{Major-axis Features}

Figure 13 shows a series of intensity slices taken at different wavelengths along the major axis of M31. The data are normalized against the peak flux in each band and are convolved to a resolution of $24^{\prime \prime}$ FWHM (the same as was used for the flux density measurements). The $500 \mu \mathrm{m}$ data have been left unconvolved as their PSF is larger than the $350 \mu \mathrm{m}$ PSF. The annotations show the positions of the arm crossing regions described by Baade (1963) from his survey of M31 using the Mount Wilson 100 -inch telescope. These are labeled numerically proceeding from the center outward in northerly and southerly directions. The innermost $\mathrm{N} 1$ and $\mathrm{S} 1$ arms, and to an extent the N2 and $\mathrm{S} 2$ crossing points, show an excess of emission at shorter wavelengths. This is the region that appears blue in the false-color image in Figure 1, indicating the presence of hot dust. The N3 crossing point shows a strong peak of emission at long wavelengths relative to the other inner arms.

Paper I reported the existence of a series of low brightness rings and structures surrounding M31 in the Herschel maps. Paper I also confirmed the detection of a $15 \mathrm{kpc}$ ring previously seen with the Infrared Space Observatory (Haas et al. 1998) and Spitzer (Gordon et al. 2006). The $15 \mathrm{kpc}$ ring (equivalent to $\sim 1^{\circ}$ at $785 \mathrm{kpc}$ ) is seen in Figure 13 as the peaks coincident with Baade's N5 and S5 arm crossing points. In addition to the $15 \mathrm{kpc}$ ring, Paper I reported three additional structures they labeled E, F, and $\mathrm{G}$ at major-axis distances of $\sim 21, \sim 26$, and $\sim 31 \mathrm{kpc}$ (equivalent to $\sim 1.5, \sim 1.9$, and $\sim 2.25$ at $785 \mathrm{kpc}$ ). The E feature is visible as the faint red band on the right of Figure 1 and a minor rise in emission associated with the S6 arm crossing in Figure 13. The S7 crossing point is not shown, but at 1.9 from the center, it would be coincident with the $\mathrm{F}$ feature in the Herschel maps.

Analysis of M31's spiral structure is hampered by the heavy disruption to the galaxy in the southern quadrant. The most significant feature is a $30^{\circ}$ wide break in the ring at a position of $(8,-8) \mathrm{kpc}$ coincident with the position of star-forming cloud NGC 206 (visible in Figure 14). There is further evidence of this disruption in the arm crossing slice shown in Figure 13. The northern arm segments are all coincident with the strongest emission peaks. However, the southern crossing points only show a weak coincidence, if any, with the strongest emission peaks. There is some emission peaking with S2 and S3, but the brightest peaks actually occur between the S3 and S4 as part of an extended plateau of emission that stretches from S2 to S5. The outermost of these two peaks, at $\sim 40^{\prime}$, is coincident with the sweep of the ring and the possible spiral arm pattern.

\subsection{Structural Parameters}

In order to more accurately describe the properties of the Andromeda spiral arms, we follow Gordon et al. (2006) and analyze the $10 \mathrm{kpc}$ ring separate from the arms themselves. 


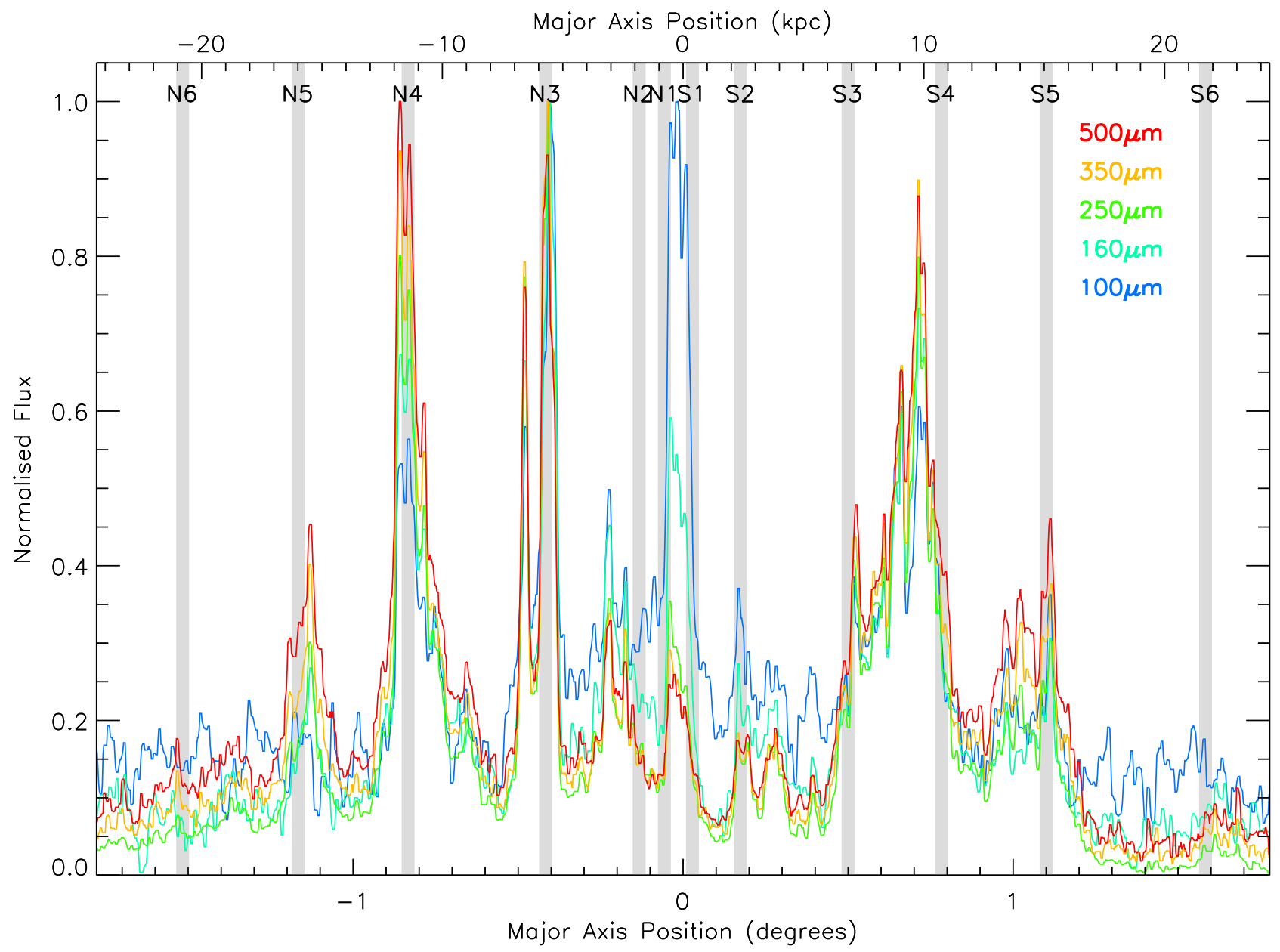

Figure 13. Normalized intensity slices along the major axis of M31 from PACS $100 \mu \mathrm{m}$ (blue) to Herschel SPIRE $500 \mu \mathrm{m}$ (red); the key in the top right shows the color assigned to each wavelength. The positions of the arm crossing points from Baade (1963) are annotated as N1-6 and S1-6.
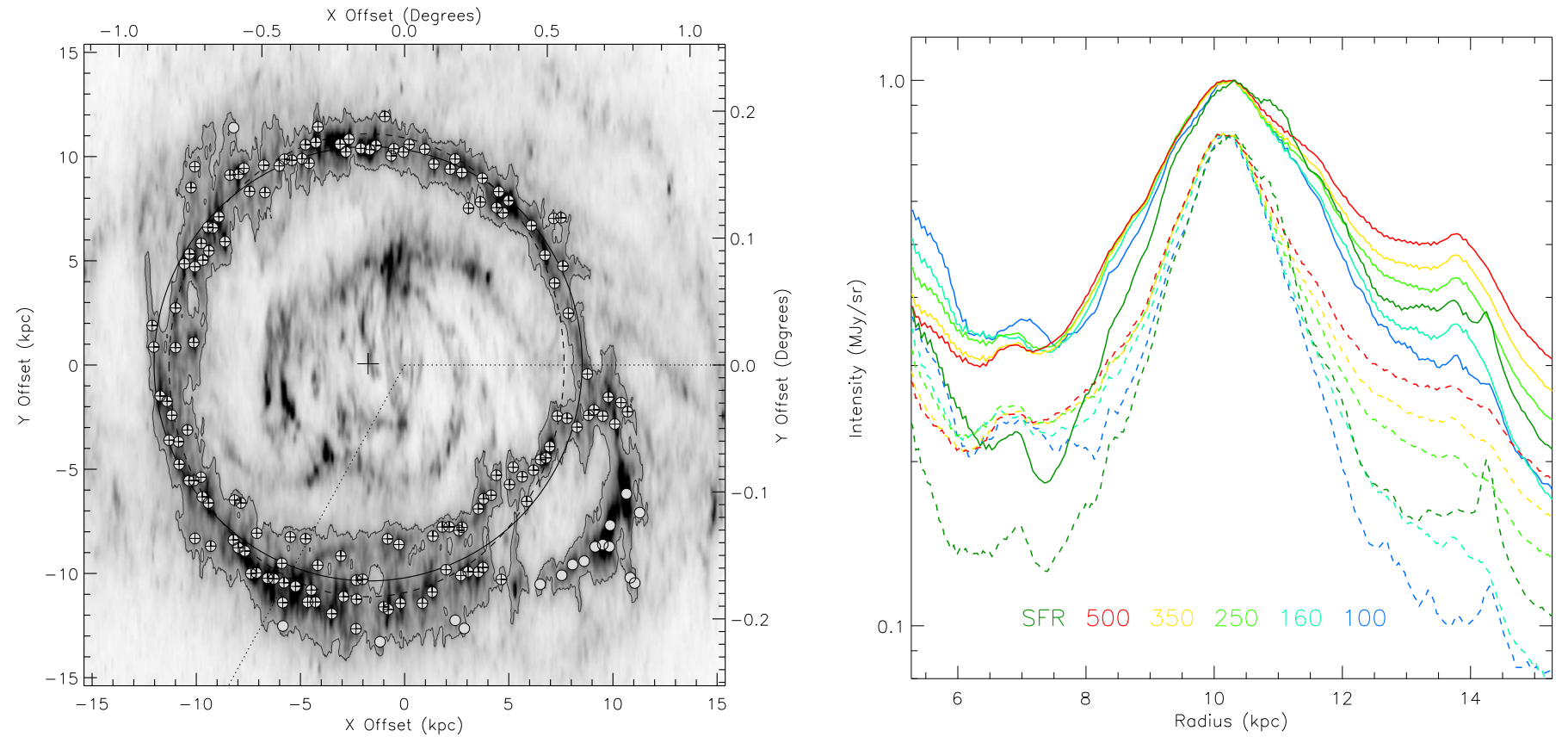

Figure 14. Left: Enlargement of the center of Andromeda. The grayscale is the deprojected SPIRE $250 \mu \mathrm{m}$ map. The black contour is the $10 \mathrm{kpc}$ ring node from Figure 3. The clouds of the ring are shown by the circle markers; those involved in the final ring fit have a cross inside the markers (see the text for details). The solid and dashed circles show the ring fitted with assumed inclination angles of $77^{\circ}$ and $75^{\circ}$, respectively. The dotted lines show the P.A. range $120^{\circ}-240^{\circ}$. Right: circularly averaged profile of the $10 \mathrm{kpc}$ ring. The solid lines show the Herschel wavelengths (see key for colors) averaged over all P.A.s. The dashed lines show the profiles excluding the data from the P.A. range $120^{\circ}-240^{\circ}$. 
Table 3

Structural Parameters for M31 by Assumed Angle of Inclination $i$

\begin{tabular}{|c|c|c|c|c|c|c|c|c|}
\hline \multirow{2}{*}{$\begin{array}{l}i \\
\left(^{\circ}\right)\end{array}$} & \multicolumn{3}{|c|}{10 kpc Ring } & \multicolumn{3}{|c|}{15 kpc Ring } & \multicolumn{2}{|c|}{ Spiral Arms } \\
\hline & $\begin{array}{c}R_{10 \mathrm{kpc}} \\
(\mathrm{kpc})\end{array}$ & Center R.A. & Center Decl. & $\begin{array}{c}R_{15 \mathrm{kpc}} \\
(\mathrm{kpc})\end{array}$ & Center R.A. & Center Decl. & $\begin{array}{c}\phi \\
\left({ }^{\circ}\right)\end{array}$ & $\begin{array}{c}a \\
(\mathrm{kpc})\end{array}$ \\
\hline 75 & 9.5 & $00^{\mathrm{h}} 51^{\mathrm{m}} 22^{\mathrm{s}} .0$ & $+42^{\circ} 35^{\prime} 14^{\prime \prime}$ & 15.5 & $00^{\mathrm{h}} 50^{\mathrm{m}} 59^{\mathrm{s}} .0$ & $+42^{\circ} 30^{\prime} 35^{\prime \prime}$ & 9.1 & 6.4 \\
\hline
\end{tabular}

Gordon et al. (2006) described the structure of M31 with a classic two-arm logarithmic spiral and an offset ring. In addition to this radial profile analysis, we attempt a fit to the $15 \mathrm{kpc}$ ring. A summary of the results is given in Table 3 .

\subsubsection{The $10 \mathrm{kpc}$ Ring}

Figure 14 (left) shows a $250 \mu \mathrm{m}$ grayscale map of the center of M31. The branch network that composes the $10 \mathrm{kpc}$ ring (as identified by the box/contour in Figure 3 ) is shown by the gray contour. The position of these GMCs is shown by the circular markers. An offset circle is fit to them in two stages: we exclude GMCs in the P.A. range $120^{\circ}-240^{\circ}$ (shown by the dotted wedge in Figure 14). This gives an initial fit to the undistributed portion of the ring.

We then repeat the fit for all ring GMCs over all P.A.s with a galactocentric radius within $1.5 \mathrm{kpc}$ of the first fit's result. These GMCs are shown by a cross inside their marker. It is the fit to these filtered GMCs that gives us the parameters for the ring. The best-fit ring is shown by the black circle on Figure 14, and its parameters are listed in Columns $2-4$ of Table 3 . The fitted center is shown by the cross and is offset from the assumed center of M31 by $1.5 \mathrm{kpc}$ along the negative $x$-axis.

The exact extent of the deprojected ring is sensitive to M31's assumed angle of inclination. This angle can be estimated by fitting position-velocity tilted ring models to molecular line data (Chemin et al. 2009; Corbelli et al. 2010) of M31. We have used the HELGA assumed inclination angle of $77^{\circ}$ to fit our best-fit radius of $10.3 \mathrm{kpc}$. This angle is based on a mean value from the (Chemin et al. 2009) model. However, in their original analysis Gordon et al. (2006) derived a radius of $9.8 \mathrm{kpc}$ using an inclination angle of $75^{\circ}$. This is closer to the mean value found in the Corbelli et al. (2010) model.

We repeated our fitting using the Gordon et al. (2006) angle of inclination and found a radius of $9.5 \mathrm{kpc}$, in excellent agreement with their value. The $9.5 \mathrm{kpc}$ fit is shown by the dashed circle in Figure 14 (it appears as an ellipse owing to the differences in the assumed inclination angles). The differing results for the two inclination angles are listed in Table 3, and the differences between the two inclination models are discussed in Appendix A.

The right panel of Figure 14 shows a radial profile of the $10 \mathrm{kpc}$ ring constructed in the coordinate frame of the $10 \mathrm{kpc}$ ring fit. Normalized flux profiles for each of the five Herschel wavelengths are shown as solid lines; the colors are the same as for Figure 13. The profiles were repeated with the exclusion of data in the P.A. range $120^{\circ}-240^{\circ}$. The second set of profiles are shown by dashed lines and have been normalized to 0.8 so as to offset them from the first set of profiles. All wavelengths shorter than $350 \mu \mathrm{m}$ have been convolved to the $350 \mu \mathrm{m}$ resolution and pixel grid.

There is a strong coincidence in the flux profiles on the interior side of the ring, all reaching a minimum at $7.5 \mathrm{kpc}$ and a maximum at $10 \mathrm{kpc}$. The correlation is particularly strong between 160 and $500 \mu \mathrm{m}$, suggesting that the cold dust component has a uniform temperature between these radii. The reverse is true on the outside of the ring, beyond $12 \mathrm{kpc}$, where the long-wavelength bands become increasingly strong. Comparison of this trend with the radial dust fits of Paper II shows that there was only a slight temperature gradient in the outer galaxy. However, there was a stronger radial trend in the dust spectral index $(\beta)$. A flattening of the Rayleigh-Jeans part of the dust SED, as shown by the changing $\beta$ profile Paper II, could explain the divergence of the flux profiles seen in Figure 14.

\subsubsection{The $15 \mathrm{kpc}$ Ring}

The existence of a $15 \mathrm{kpc}$ ring visible in infrared maps of M31 has been noted before (Haas et al. 1998; Gordon et al. 2006). Features associated with this $15 \mathrm{kpc}$ ring are visible in the Herschel maps (e.g., Figure 2) and are seen as an enhancement in the number density of molecular clouds at that radii (see Figure 6). It is reasonable then to investigate whether this ring can be fit in the same manner as the $10 \mathrm{kpc}$ ring. We do not have a unique tree branch for this structure as there is not a constant valley between it and the $10 \mathrm{kpc}$ ring.

To get around this, we select all the GMCs with a galactocentric distance larger than $14 \mathrm{kpc}$ (to exclude the $10 \mathrm{kpc}$ ring and NGC 206) and less than $18 \mathrm{kpc}$ (to exclude the outer arcs) and fit them with an offset circle. The results of the $15 \mathrm{kpc}$ fit are listed for both angles of inclination in Table 3 . The clouds associated with the best fit are colored orange in Figures 15 and 16. Both fits give equivalent radii but have slightly different centers. This exercise is only an aid to estimating the radius of this feature. Nevertheless, the $15.5 \mathrm{kpc}$ ring does appear to closely match the distribution of GMCs. It also passes through the Baade (1963) N5 and S5 arm crossing regions. The reduced $\chi^{2}$ value for this fit is $\sim 0.8$, indicating that the fit is not unreasonable even if it is slightly overconstrained (as would be expected from the filtering).

Gordon et al. (2006) investigated the offset of the $10 \mathrm{kpc}$ ring by modeling M31's interaction with the satellite galaxy M32. They showed that the passage of M32 could have triggered a wave of star formation that forms the ring itself. However, M32's proximity also causes the ring to be slightly pulled off-center, thus creating the observed offset. The $x y$ offsets we find for the $10 \mathrm{kpc}$ and $15 \mathrm{kpc}$ rings are $(-1.5,-0.3) \mathrm{kpc}$ and $(-0.3$, $-0.3) \mathrm{kpc}$, respectively. While different, both offsets do pull toward the negative- $x$, negative- $y$ direction, suggesting that they are concentric with each other.

\subsubsection{The Spiral Arms}

The "spiral arms" shown in Figure 2 are not contiguous and appear to be composed of a series of disjointed arm segments. In order to better describe these arms, we use the positions of the GMCs from our source extraction. These are filtered to remove GMCs that are within $1.5 \mathrm{kpc}$ of either of the fitted 


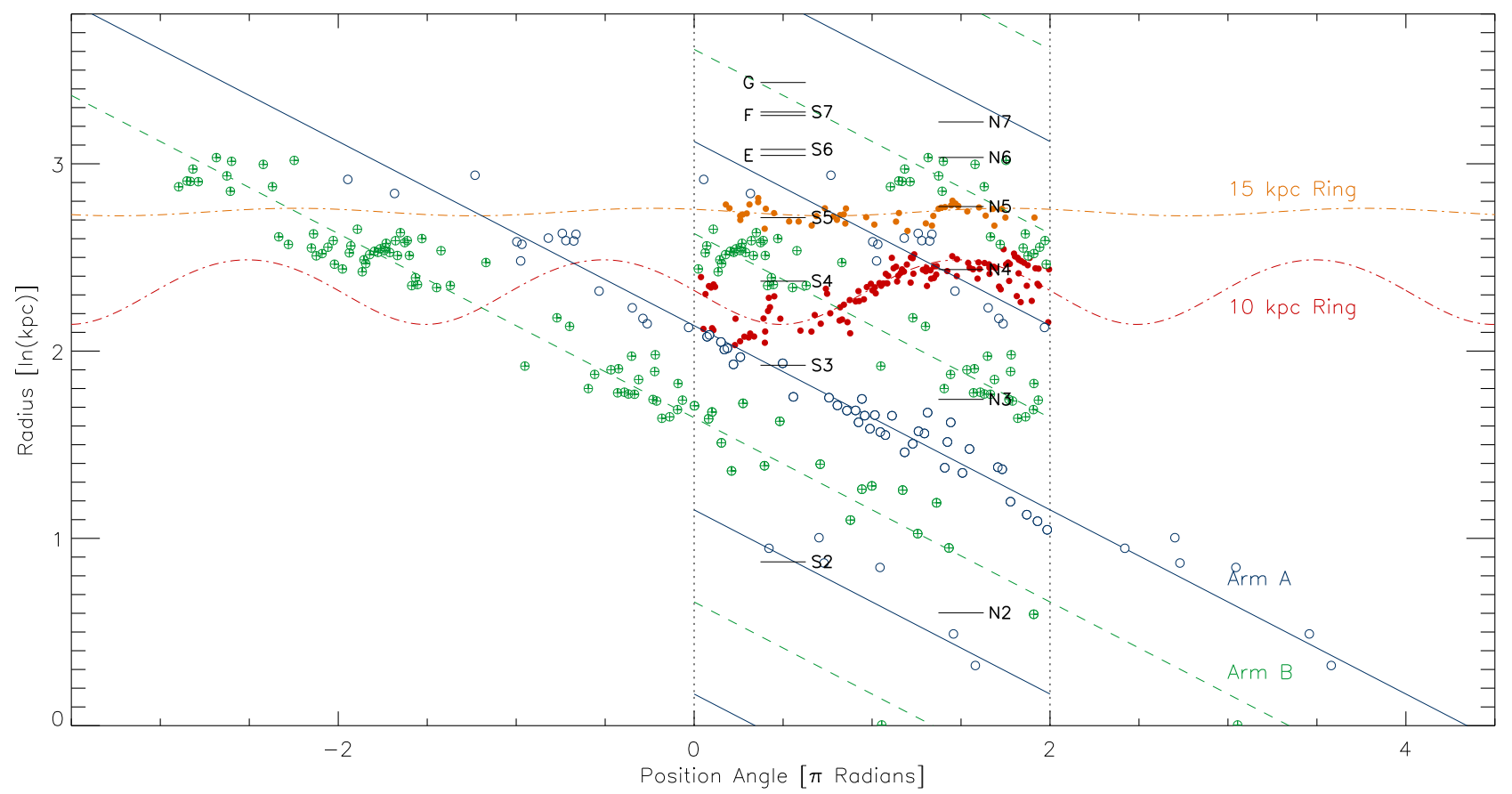

Figure 15. Polar coordinate plot of M31 GMCs. All GMCs are shown for a single $0-2 \pi$ radian range. GMCs used to fit the 10 or $15 \mathrm{kpc}$ ring are shown by solid markers. The offset circle fits to the rings are shown by the dot-dashed sine waves. GMCs used to fit the spiral arms are shown by the open circles. The GMCs associated with Arm B are differentiated from those associated with Arm A by a cross inside their marker. The best-fit logarithmic spirals are shown by the solid line (Arm A) and the dashed line (Arm B). These arm fits are replicated in the $0-2 \pi$ radian range to show how they wrap. Additionally, for each arm we unwrap and replicate the GMCs associated with it to show the full fit. The arms have the same pitch angle and are a rotation of one another. The Baade (1963) arm crossing regions are annotated. The E, F, and G features reported by Paper I are shown by the short horizontal lines. In the online version of this image the features associated with Arm A, Arm B, the $10 \mathrm{kpc}$ ring, and the $15 \mathrm{kpc}$ ring are color-coded green, blue, red, and orange, respectively.

10 or $15 \mathrm{kpc}$ rings. We then use a preliminary by-eye fit to divide the remaining GMCs into those associated with one or other of two spiral arms. The sources associated with each arm are shown plotted using polar coordinates in Figure 15 and using $X Y$-offset coordinates in Figure 16. Figure 15 is split into two regions. The first region is wrapped over the range $0-2 \pi$ radians (bracketed by the dotted lines) and is where all the GMCs are plotted with their P.A.s. Within this range the GMCs used to fit each of the 10 and $15 \mathrm{kpc}$ rings are shown by the solid markers, and the fits to the rings are shown by the dot-dashed lines.

For ease of reference we assign designations to each arm. We refer to the first arm as "Arm A" and plot its clouds as open circles. This arm passes through the southern Baade (1963) arm crossing points (S2 and S3) interior to the $10 \mathrm{kpc}$ and then crosses the ring close to N4. We refer to the second arm as "Arm B" and plot the clouds associated with it as circles with crosses. Arm B passes through the Baade (1963) arm crossing points (N3 and S4) on either side of the ring. The two sets of GMCs appear to follow the same trend and suggest that they are merely a rotation of one another.

The clouds in Figure 15 are plotted with (full $\theta$ range) and without ( $\theta$ wrapped to $0-\pi$ radians) their phase shift removed. Classic logarithmic spiral arms show up as linear features on a plot of $\ln (r)$ versus $\theta$. The equation for these can be written in the form $r=a \exp (b \theta)$, where $r$ and $\theta$ are the position of the spiral in polar coordinates, $a$ is a reference radius determining the relative rotation of the spiral, and $b$ is a constant related to the pitch angle $\phi$ by $b=1 / \tan (90-\phi)$. A linear regression was performed on the unwrapped arm GMCs under the assumption that they had a common pitch angle and were offset from each other by $180^{\circ}$. The results are listed in Table 3 . Repeating the fit without excluding the $15 \mathrm{kpc}$ ring GMCs or fitting the arms individually gave values that were within the errors on the original pitch-angle fit $\left( \pm 0.14\right.$ for $\left.i=77^{\circ}\right)$. The $i=75^{\circ}$ fit is within $3 \sigma$ of the pitch angle $(i=9.5)$ found by Gordon et al. (2006).

Also plotted in Figure 15 are the positions of the Baade (1963) arm crossing regions and the features E, F, and G from Paper I. Features $\mathrm{E}$ and $\mathrm{F}$ are associated with regions $\mathrm{S} 6$ and $\mathrm{S7}$, but neither N6, N7, S6, nor S7 appears to be closely associated with any particular arm. It is possible that evolution in the arm's pitch angle or changes in inclination angle are influencing features this far out. We explore the effects of a nonuniform inclination angle on M31's spiral structure in Appendix A.

There is a disrupted portion of the ring at $X=8, Y=-8 \mathrm{kpc}$ that appears to be an interarm hole cleared out between the two spiral arms. Gordon et al. (2006) simulated the interaction of M32 with M31's disk and showed that such a hole could be created by M32's passage through the disk. The survival of this hole against differential rotation implies a relatively short timescale, on the order of $20 \mathrm{Myr}$ (Gordon et al. 2006). A general effect of M32's passage could have been a wave of star formation within M31's disk (Gordon et al. 2006). M32's H iI luminosity function is double peaked, with the fainter peak being consistent with emission from a population of the B stars with a lifetime of $15 \mathrm{Myr}$ (Azimlu et al. 2011), comparable to the timescale for the passage of M32 through the M31 disk.

\section{SUMMARY}

We have used HELGA (Paper I) data taken with the Herschel Space Observatory (wavelengths $100-500 \mu \mathrm{m}$ ) to create a catalog of molecular clouds in the nearby galaxy of Andromeda.

1. Monochromatic source extraction was performed on the M31 field using the hierarchical source extraction algorithm CSAR (Kirk et al. 2013). The tree of sources was 


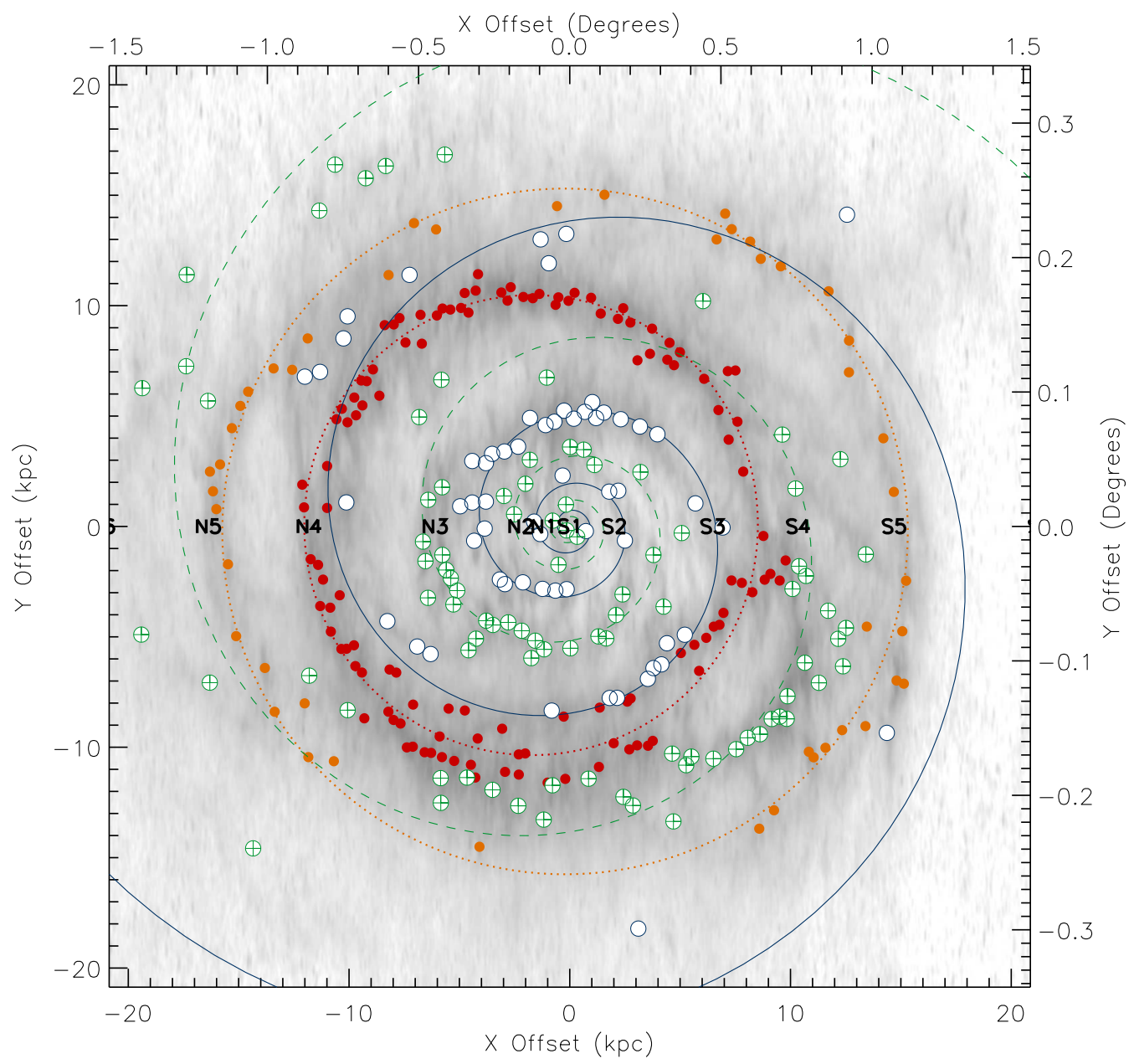

Figure 16. M31's spiral arms. The 10 and $15 \mathrm{kpc}$ rings are shown by dotted circles. GMCs associated with the arms are shown by open markers; GMCs associated with Arm B are differentiated by the cross over their marker. The best-fit logarithmic spirals are shown by the solid line (Arm A) and the dashed line (Arm B). The Baade (1963) arm crossing regions are annotated. In the online version of this image the features associated with Arm A, Arm B, the $10 \mathrm{kpc}$ ring, and the $15 \mathrm{kpc}$ ring are color-coded green, blue, red, and orange, respectively.

pruned back to that containing the contiguous emission from M31 alone. A total of 651 nodes were found in the structure tree. Of these, 326 were leaf nodes, i.e., sources without resolved substructure. These are the sources that form the catalog presented in this paper.

2. The surface number density of clouds peaks toward the center of M31 and falls off at a rate similar to that of the optical surface brightness out to $15 \mathrm{kpc}$. On top of this distribution are a series of peaks at $\sim 5,10$, and $15 \mathrm{kpc}$ coincident with the reported rings of emission at several of those wavelengths. In addition, Paper I found a series of arc-like features at $\sim 20,25$, and $30 \mathrm{kpc}$, suggesting that M31 contains a set of nested weak resonant rings whose radii are multiples of $5 \mathrm{kpc}$.

3. Herschel photometry was performed for each of the clouds. The temperature and mass of each cloud were found by fitting a gray body to its SED. The dust parameters were described by the radial dust relationships (dust-to-mass ratio, dust emissivity) from Paper II. The median dust temperature was $18 \mathrm{~K}$.

4. Clouds with masses in the range $10^{4}-10^{7} M_{\odot}$ and with sizes of 100-1000 pc were found. This and a comparison with interferometry maps showed that we are resolving structures that are comparable to large GMCs and complexes of multiple GMCs within the Milky Way. The power-law slope of the cloud's cumulative mass function agreed with that found in other extragalactic studies of molecular clouds and in interferometric studies of M31.

5. The clouds' properties appear to be consistent with those of clouds found in the Milky Way. Specifically, the FIR luminosity function, the relationship of FIR to mid-infrared luminosity, and the relationship of cloud mass to ${ }^{12} \mathrm{CO}$ luminosity are all consistent with that found for clouds in the Milky Way. The last relationship was found to be virtually identical to that found by Solomon et al. (1987) for clouds in the Milky Way.

6. Following Gordon et al. (2006), we fit an offset circle to the dominant ring feature and calculate a radius of $10.3 \mathrm{kpc}$. Our results were consistent with Gordon et al. (2006), allowing for differences in assumed inclination angle. We also fit an offset circle to the clouds at $15 \mathrm{kpc}$ and derive a radius of $15.5 \mathrm{kpc}$. The centers of both rings are offset in the same approximate direction from the assumed center of M31.

7. Clouds associated with the 10 and $15 \mathrm{kpc}$ rings were excluded, and a logarithmic spiral was fit to the remaining sources. A common pitch angle of 8.9 was found for two spiral arms that trailed one another by $180^{\circ}$. The fitted arms and rings are consistent with the arm crossing features described by Baade (1963). 


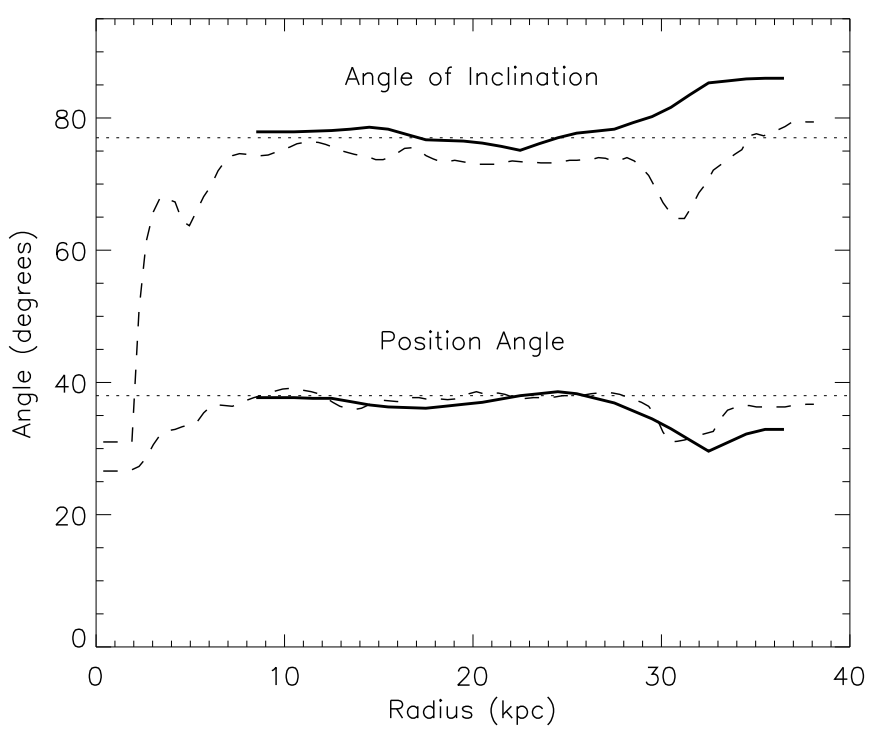

Figure 17. Inclination angles (top) and P.A.s (bottom) from the Chemin (dashed lines) and Corbelli (solid lines) tilted-ring models. The dotted lines show the values adopted by HELGA.

SPIRE has been developed by a consortium of institutes led by Cardiff University (UK) and including Univ. Lethbridge (Canada); NAOC (China); CEA, LAM (France); IFSI, Univ. Padua (Italy); IAC (Spain); Stockholm Observatory (Sweden); Imperial College London, RAL, UCL-MSSL, UKATC, Univ. Sussex (UK); and Caltech, JPL, NHSC, Univ. Colorado (USA). This development has been supported by national funding agencies: CSA (Canada); NAOC (China); CEA, CNES, CNRS (France); ASI (Italy); MCINN (Spain); Stockholm Observatory (Sweden); STFC (UK); and NASA (USA).

PACS has been developed by a consortium of institutes led by MPE (Germany) and including UVIE (Austria); KU Leuven, CSL, IMEC (Belgium); CEA, LAM (France); MPIA (Germany); INAFIFSI/OAA/OAP/OAT, LENS, SISSA (Italy); and IAC (Spain). This development has been supported by the funding agencies BMVIT (Austria), ESA-PRODEX (Belgium), CEA/CNES (France), DLR (Germany), ASI/INAF (Italy), and CICYT/MCYT (Spain).

\section{APPENDIX A}

\section{DEPROJECTION}

It has been known since the earliest studies of Andromeda's structure (Baade 1963; Arp 1964) that its stellar disk exhibited a pronounced warp at large radii. This warp means that studies that use a constant position and inclination angle to deproject M31 are liable to introduce artifacts resulting from differences between the assumed flat geometry and the actual warped geometry. In order to quantify possible problems of this sort, we study the effects of projecting M31 using two recent models published by Chemin et al. (2009, hereafter the Chemin model) and Corbelli et al. (2010, hereafter the Corbelli model) using independent H I surveys. Direct comparison of the models is made easier as both use the same distance to Andromeda as adopted by the HELGA consortium (McConnachie et al. 2005).

The two literature models analyzed M31 as a series of nested, tilted rings. Each ring represents the projection of a particular circular orbit with its own angle of inclination $i(R)$ and P.A. $\theta(R)$. Figure 17 plots the inclination and P.A.s from Table 4 of
Chemin et al. (2009) and Table 1 of Corbelli et al. (2010). The Chemin model tabulated parameters for the entire disk from the center to $38 \mathrm{kpc}$, but the Corbelli model only tabulated values over the range $8.5-36.5 \mathrm{kpc}$ as they did not model the inner part of the Hi disk. The Corbelli model includes small offsets $\left(x_{0}, y_{0}\right)$ of an order of $1^{\prime}-2^{\prime}$ (less than $\left.0.5 \mathrm{kpc}\right)$ to the central position of each ring, but the Chemin model does not.

Figure 17 shows that the P.A.s adopted by the two models broadly agree. However, the angle of inclination adopted by the Corbelli model is systematically higher than the value adopted by the Chemin model. The mean inclination angle over the range $10-20 \mathrm{kpc}$ is $75^{\circ} \pm 1^{\circ}$ for the Chemin model and $77^{\circ} \pm 1.0$ for the Corbelli model (this is the value assumed by the HELGA survey). Likewise, the mean P.A. over the same range is $37.5 \pm 0.9$ for the Chemin model and $37.3 \pm 0.8$ for the Corbelli model. These inclination angles represent a deprojection factor of $\times 3.86$ for the Chemin model and $\times 4.44$ for the Corbelli model along the projected minor axis, a difference of approximately $15 \%$. The models also differ in their trends in the outer disk, where the warp should be most noticeable. Both models include a P.A. minimum at around $\sim 32 \mathrm{kpc}$. The Chemin model includes a similar drop in the inclination angle, but the Corbelli model does not and has the inclination angle increasing monotonically in the outer disk.

To examine the effects of these two models, we construct a simple toy model of M31 consisting of a series of concentric circular rings and twin logarithmic spiral arms with a pitch angle of 8.5. The top row of Figure 18 shows the effects of projecting the toy model onto the plane of the sky using the constant angles assumed by HELGA $\left(i=77^{\circ}, \theta=38^{\circ}\right)$, the Corbelli model, and the Chemin model. For the flat (constant angles) geometry the inclination of the disk means that the rings along the projected minor axis become very close but never overlap. By comparison, after $\sim 27 \mathrm{kpc}$, the Corbelli and Chemin models deviate from the approximately linear trend of P.A. with radius. This causes the rings to precess against one another and to overlap.

The P.A. trend is amplified by the aforementioned divergence of the adopted inclination angle. The effect of this is to send the outer parts of the spiral arms in opposite directions. The Corbelli model causes the outer rings and the outer spiral arm segments to be projected inward over/below the central part of the galaxy. By contrast, the Chemin model causes those same spiral arms to flare outward along the minor axis. There are also differences in the center of the galaxy as the Chemin model causes the inner part of the spiral arms to merge into a ring-like structure. No projection data were given for the Corbelli model within $8.5 \mathrm{kpc}$, so we used the data from that radius for the interior portion.

The effects of naively deprojecting warped structures while using a nonwarped assumption are explored in the middle row of Figure 18. Here the projected models from the top row are deprojected using the constant position and inclination angles used for the first column. As expected, the first column deprojects perfectly, but there are significant artifacts introduced into the other two panels. The assumed $i$ and $\theta$ most closely match the Corbelli model, so it is unsurprising that it appears the most circular. This exercise reinforces how structure assumed to be at one radius, particularly faint structure as would be found on the tail end of a spiral arm, may actually be at a completely different radius.

The bottom line of panels shows the $350 \mu \mathrm{m}$ map of M31 (the one used for source identification) resampled into a rectilinear face-on grid under the assumptions of the flat geometry, 


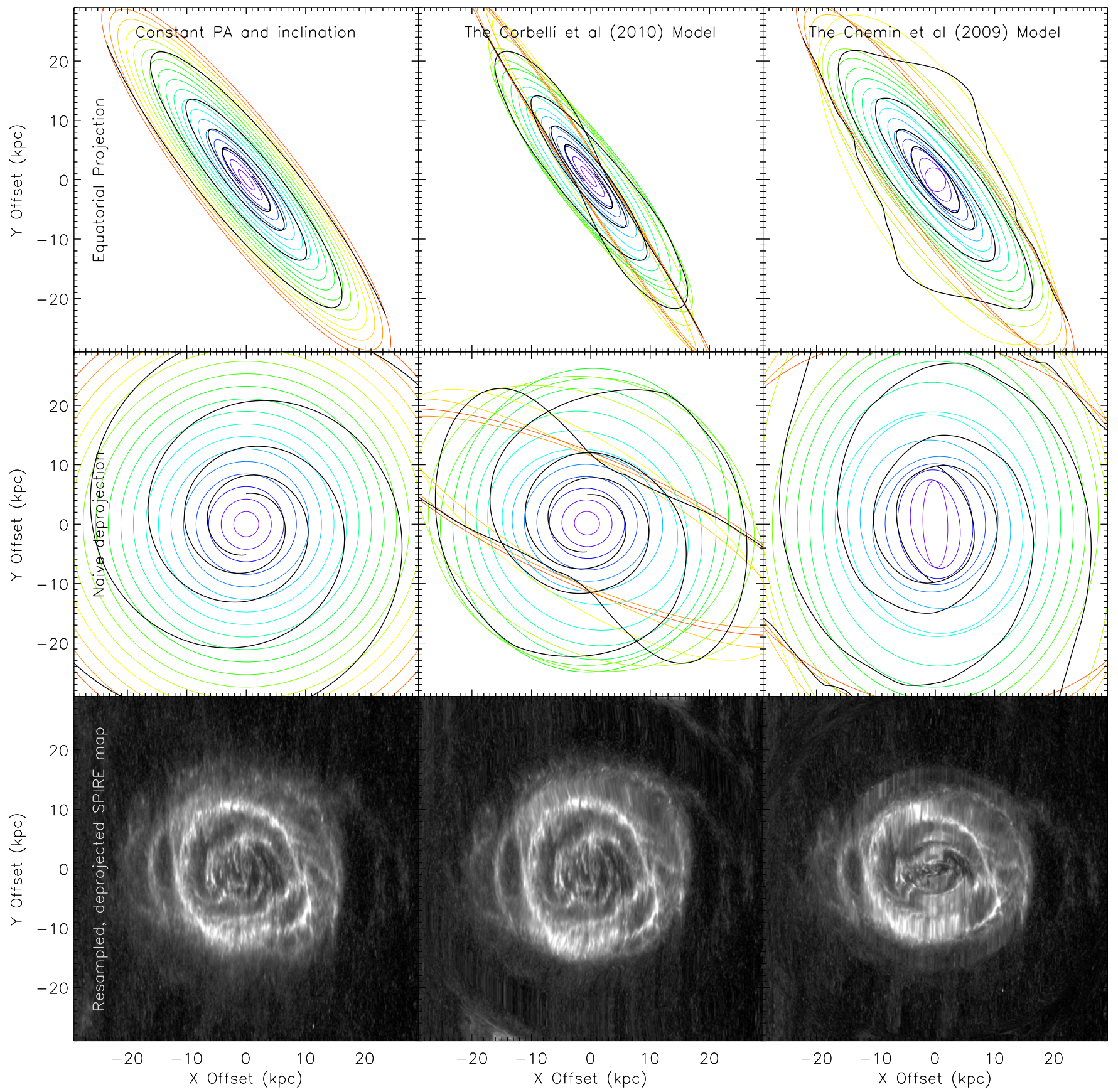

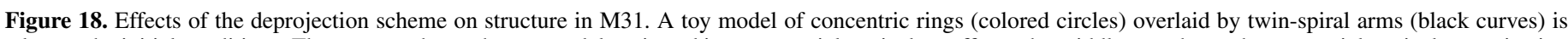

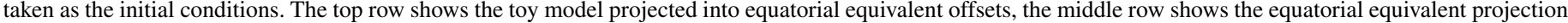

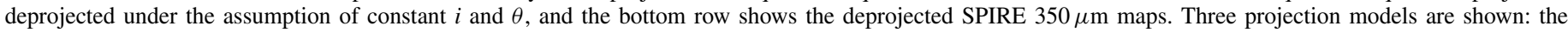
left-hand column shows the results of using constant $i$ and $\theta$, the middle column shows the Corbelli model, and the right-hand column shows the Chemin model.

Corbelli, and Chemin models. These maps were created by calculating the right ascension and declination for every pixel under the assumptions of each model. Each pixel was then assigned the brightness of the original map at that right ascension and declination. For the constant-angle example each $x, y$ pixel mapped uniquely onto a single R.A., decl. position. However, there was a degeneracy in the tilted ring models where multiple $x, y$ pixels mapped onto the same R.A., decl. position, as would be expected from the overlapping rings in the preceding panels. This effect created regions on the deprojected maps where features were stretched out and blurred.
Comparison of the deprojected $350 \mu \mathrm{m}$ map in Figure 18 with the other panels shows that the majority of emission is within a radius of $\sim 22 \mathrm{kpc}$, and this is not directly affected by the strongest parts of the outer warp. Indeed, the warp only becomes important when the Corbelli model projects spiral arms over the center of the galaxy. Of the two variable $i, \theta$ models it is the Chemin model that gives a version of M31 that appears the most circular at large radii. However, it also displays significant degeneracy along the projected short axis. It does, unlike the Corbelli model, deproject the center of the galaxy. The lozenge/bar-shaped inner structure shown in the middle 


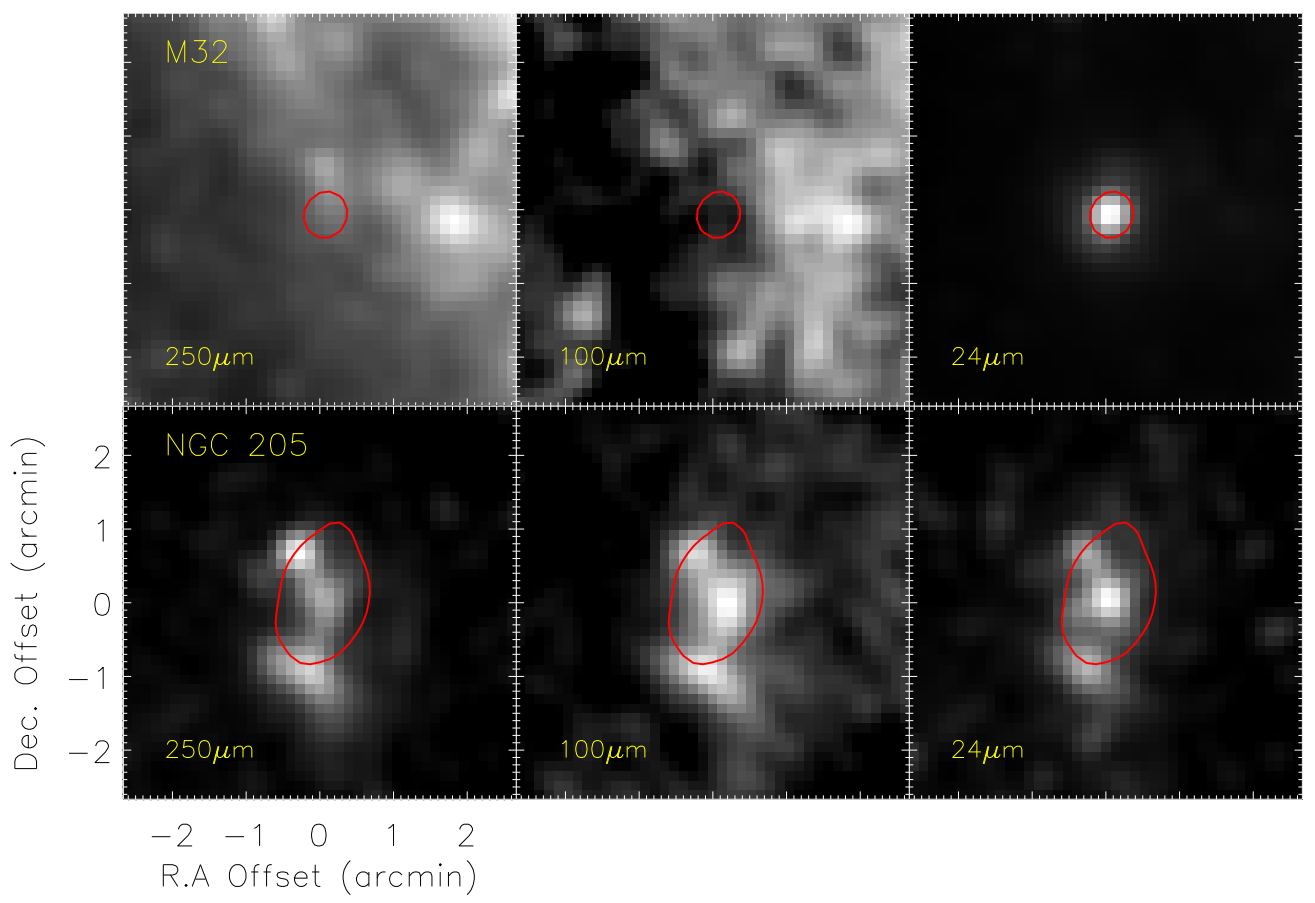

Figure 19. Two of the satellites of Andromeda, M32 and NGC 205, showing markedly different emission properties with wavelength. The individual wavelengths are labeled. The data have been convolved to the $350 \mu \mathrm{m}$ resolution. The IRAC $3.6 \mu \mathrm{m} 50 \%$ flux contour is shown in red. The position center for M32 is $0^{\mathrm{h}} 42^{\mathrm{m}} 41.87$, $40^{\circ} 51^{\prime} 57.2^{\prime \prime} .50$ and for NGC 205 is $0^{\mathrm{h}} 40^{\mathrm{m}} 00^{\mathrm{s}} .08,41^{\circ} 41^{\prime} 07.1^{\prime \prime} .50$.

right panel of Figure 18 is similar to that seen in the red giant branch image shown in Figure 1.

\section{APPENDIX B \\ SATELLITES}

There are two dwarf galaxies within the M31 field, M32 and NGC 205, which are of note for their dramatically varying dust emission. Figure 19 shows both galaxies at SPIRE $350 \mu \mathrm{m}$, PACS $100 \mu \mathrm{m}$, and Spitzer MIPS $24 \mu \mathrm{m}$; the contour shows the Spitzer IRAC $3.6 \mu \mathrm{m} 50 \%$ peak intensity contour. All the data have been convolved to the $350 \mu \mathrm{m}$ resolution. M32 appears strongly at $24 \mu \mathrm{m}$ but is completely devoid of emission in the Herschel images. By contrast, NGC 205 shows revolved emission at three positions - a central peak coincident with short-wavelength center and long-wavelength peaks to the north and south of it. See De Looze et al. (2012) for a study of the NGC 205 data.

\section{REFERENCES}

Allen, R. J., Le Bourlot, J., Lequeux, J., Pineau des Forets, G., \& Roueff, E. 1995, ApJ, 444, 157

Aniano, G., Draine, B. T., Gordon, K. D., \& Sandstrom, K. 2011, PASP, 123,1218

Arp, H. 1964, ApJ, 139, 1045

Asplund, M., Grevesse, N., Sauval, A. J., \& Scott, P. 2009, ARA\&A, 47, 481

Azimlu, M., Marciniak, R., \& Barmby, P. 2011, AJ, 142, 139

Baade, W. 1963, Evolution of Stars and Galaxies, ed. C. H. P. Gaposchkin (Cambridge, MA: Harvard Univ. Press)

Barnard, E. E. 1919, ApJ, 49, 1

Bendo, G. J. 2011, SPIRE Photometry Cookbook (revision 2011 July 8) Technical Report, SPIRE-ICC

Bernard, J.-P., Paradis, D., Marshall, D. J., et al. 2010, A\&A, 518, L88

Blitz, L., Fukui, Y., Kawamura, A., et al. 2007, Protostars and Planets V (Tucson, AZ: Univ. Arizona Press), 81

Boulanger, F., Combes, F., \& Stark, A. A. 1981, A\&A, 93, L1

Burstein, D., \& Heiles, C. 1982, AJ, 87, 1165

Buta, R. 1999, Ap\&SS, 269, 79
Bystedt, J. E. V., Brinks, E., de Bruyn, A. G., et al. 1984, A\&AS, 56, 245 Calzetti, D., Kennicutt, R. C., Engelbracht, C. W., et al. 2007, ApJ, 666, 870 Carey, S. J., Noriega-Crespo, A., Mizuno, D. R., et al. 2009, PASP, 121, 76 Chemin, L., Carignan, C., \& Foster, T. 2009, ApJ, 705, 1395 Churchwell, E., Babler, B. L., Meade, M. R., et al. 2009, PASP, 121, 213 Corbelli, E., Lorenzoni, S., Walterbos, R., Braun, R., \& Thilker, D. 2010, A\&A, 511, A89

Courteau, S., Widrow, L. M., McDonald, M., et al. 2011, ApJ, 739, 20 Dame, T. M., Hartmann, D., \& Thaddeus, P. 2001, ApJ, 547, 792

De Looze, I., Baes, M., Parkin, T. J., et al. 2012, MNRAS, 423, 2359 Devereux, N. A., Price, R., Wells, L. A., \& Duric, N. 1994, AJ, 108, 1667 Draine, B. T. 2003, ARA\&A, 41, 241

Eales, S., Smith, M. W. L., Auld, R., et al. 2012, ApJ, 761, 168

Ford, G. P., Gear, W. K., Smith, M. W. L., et al. 2013, ApJ, 769, 55

Fritz, J., Gentile, G., Smith, M. W. L., et al. 2012, A\&A, 546, A34

Fukui, Y., \& Kawamura, A. 2010, ARA\&A, 48, 547

Goodman, A. A., Rosolowsky, E. W., Borkin, M. A., et al. 2009, Natur, 457, 63 Gordon, K. D., Bailin, J., Engelbracht, C. W., et al. 2006, ApJL, 638, L87

Gouliermis, D. A., Schmeja, S., Klessen, R. S., de Blok, W. J. G., \& Walter, F. 2010, ApJ, 725, 1717

Gratier, P., Braine, J., Rodriguez-Fernandez, N. J., et al. 2012, A\&A, 542, A108 Griffin, M. J., Abergel, A., Abreu, A., et al. 2010, A\&A, 518, L3

Haas, M., Lemke, D., Stickel, M., et al. 1998, A\&A, 338, L33 Habing, H. J., Miley, G., Young, E., et al. 1984, ApJL, 278, L59

Harris, S., \& Clegg, P. E. 1983, MNRAS, 203, 955

Hodge, P. W. 1980, AJ, 85, 376

Houlahan, P., \& Scalo, J. 1992, ApJ, 393, 172

Hughes, A., Wong, T., Ott, J., et al. 2010, MNRAS, 406, 2065

Ibata, R., Chapman, S., Ferguson, A. M. N., et al. 2005, ApJ, 634, 287

Jackson, J. M., Finn, S. C., Rathborne, J. M., Chambers, E. T., \& Simon, R. 2008, ApJ, 680, 349

Jungwiert, B., \& Palous, J. 1996, A\&A, 311, 397

Kennicutt, R. C., \& Evans, N. J. 2012, ARA\&A, 50, 531

Kennicutt, R. C., Jr. 1998a, ARA\&A, 36, 189

Kennicutt, R. C., Jr. 1998b, ApJ, 498, 541

Kirk, J. M., Ward-Thompson, D., Palmeirim, P., et al. 2013, MNRAS, 432, 1424 Lada, C. J., Margulis, M., Sofue, Y., Nakai, N., \& Handa, T. 1988, ApJ, 328,143

Lee, E. J., Murray, N., \& Rahman, M. 2012, ApJ, 752, 146

Loinard, L., \& Allen, R. J. 1998, ApJ, 499, 227

Lynds, B. T. 1962, ApJS, 7, 1 
Markwardt, C. B. 2009, in ASP Conf. Ser. 411, Astronomical Data Analysis Software and Systems XVIII, ed. D. A. Bohlender, D. Durand, \& P. Dowler (San Francisco, CA: ASP), 251

Maschberger, T., \& Kroupa, P. 2009, MNRAS, 395, 931

McConnachie, A. W., Irwin, M. J., Ferguson, A. M. N., et al. 2005, MNRAS, 356, 979

Molinari, S., Swinyard, B., Bally, J., et al. 2010, PASP, 122, 314

Müller, T., Nielbock, M., Balog, Z., et al. 2011, PACS Photometer - Point-Source Flux Calibration (revision 2011 April 12, Technical Report PICC-ME-TN07, PACS-ICC

Neininger, N., Guelin, M., Garcia-Burillo, S., Zylka, R., \& Wielebinski, R. 1996, A\&A, 310, 725

Nieten, C., Neininger, N., Guélin, M., et al. 2006, A\&A, 453, 459

Paladini, R., Linz, H., Altieri, B., \& Ali, B. 2012, Assessment Analysis of the Extended Emission Calibration for the PACS Red Channel, Technical Report PICC-NHSC-TR-034, v1.0, Herschel Science Centre

Pilbratt, G. L., Riedinger, J. R., Passvogel, T., et al. 2010, A\&A, 518, L1

Planck Collaboration, Abergel, A., Ade, P. A. R., et al. 2011, A\&A, 536, A25

Poglitsch, A., Waelkens, C., Geis, N., et al. 2010, A\&A, 518, L2

Reid, M. J., Menten, K. M., Zheng, X. W., et al. 2009, ApJ, 700, 137

Robitaille, T. P., \& Whitney, B. A. 2010, ApJL, 710, L11

Roman-Duval, J., Jackson, J. M., Heyer, M., Rathborne, J., \& Simon, R. 2010, ApJ, 723, 492

Rosolowsky, E. 2007, ApJ, 654, 240
Rosolowsky, E., Keto, E., Matsushita, S., \& Willner, S. P. 2007, ApJ, 661, 830 Rosolowsky, E. W., Pineda, J. E., Kauffmann, J., \& Goodman, A. A. 2008, ApJ, 679, 1338

Sanders, D. B., Scoville, N. Z., \& Solomon, P. M. 1985, ApJ, 289, 373

Schmidt, M. 1959, ApJ, 129, 243

Sheth, K., Vogel, S. N., Wilson, C. D., \& Dame, T. M. 2000, in Proceedings 232. WE-Heraeus Seminar, ed. E. M. Berkhuijsen, R. Beck, \& R. A. M. Walterbos (Aachen: Shaker), 37

Sheth, K., Vogel, S. N., Wilson, C. D., \& Dame, T. M. 2008, ApJ, 675, 330

Skrutskie, M. F., Cutri, R. M., Stiening, R., et al. 2006, AJ, 131, 1163

Smith, M. W. L., Eales, S. A., Gomez, H. L., et al. 2012, ApJ, 756, 40

Solomon, P. M., Rivolo, A. R., Barrett, J., \& Yahil, A. 1987, ApJ, 319, 730

Solomon, P. M., Sanders, D. B., \& Scoville, N. Z. 1979, in IAU Symp. 84, The Large-Scale Characteristics of the Galaxy, ed. W. B. Burton (Cambridge: Cambridge Univ. Press), 35

Valtchanov, I. 2011, SPIRE Observers' Manual, Technical Report HERSCHELDOC-0798, v2.4, Herschel Science Centre

Vogel, S. N., Boulanger, F., \& Ball, R. 1987, ApJL, 321, L145

Vutisalchavakul, N., \& Evans, N. J., II 2013, ApJ, 765, 129

Watkins, L. L., Evans, N. W., \& An, J. H. 2010, MNRAS, 406, 264

Wilcock, L. A., Ward-Thompson, D., Kirk, J. M., et al. 2012, MNRAS, 422, 1071

Wilson, C. D., \& Rudolph, A. L. 1993, ApJ, 406, 477

Wünsch, R., Jáchym, P., Sidorin, V., et al. 2012, A\&A, 539, A116 